\title{
Error Analysis and Compensation of Gyrocompass Alignment for SINS on Moving Base
}

\author{
Bo Xu, ${ }^{1}$ Yang Liu, ${ }^{1}$ Wei Shan, ${ }^{1}$ Yi Zhang, ${ }^{2,3}$ and Guochen Wang ${ }^{1}$ \\ ${ }^{1}$ Harbin Engineering University, 145 Nantong Road, Harbin 150001, China \\ ${ }^{2}$ Beijing Aerospace Automatic Control Institute, No. 50 Yongding Road, Haidian District, Beijing 100039, China \\ ${ }^{3}$ National Key Laboratory of Science and Technology on Aerospace Intelligence Control, Beijing 100854, China
}

Correspondence should be addressed to Bo Xu; xubocarter@sina.com

Received 29 January 2014; Accepted 24 May 2014; Published 25 June 2014

Academic Editor: Bin Jiang

Copyright (C) 2014 Bo Xu et al. This is an open access article distributed under the Creative Commons Attribution License, which permits unrestricted use, distribution, and reproduction in any medium, provided the original work is properly cited.

\begin{abstract}
An improved method of gyrocompass alignment for strap-down inertial navigation system (SINS) on moving base assisted with Doppler velocity $\log$ (DVL) is proposed in this paper. After analyzing the classical gyrocompass alignment principle on static base, implementation of compass alignment on moving base is given in detail. Furthermore, based on analysis of velocity error, latitude error, and acceleration error on moving base, two improvements are introduced to ensure alignment accuracy and speed: (1) the system parameters are redesigned to decrease the acceleration interference and (2) a data repeated calculation algorithm is used in order to shorten the prolonged alignment time caused by changes in parameters. Simulation and test results indicate that the improved method can realize the alignment on moving base quickly and effectively.
\end{abstract}

\section{Introduction}

Initial alignment is the process of determining the axes orientation of strap-down inertial navigation system (SINS) with respect to the reference navigational frame. To meet the quick response of ships and enhance survivability, alignment on moving base has become a key technique for SINS [1]. Different from the alignment for SINS on static base, external information should be brought in to assist alignment for SINS on moving base [2]. At present, the research of alignment on moving base mainly focuses on the assist of GPS location. However, GPS system may have some restrictions in practical application [3-5]. Compared with GPS, Doppler velocity log (DVL) is an underwater available, independent, and high accuracy velocity measuring element commonly used on ships, and the research of initial alignment for SINS on moving base assisted with DVL has attracted much more attention [6].

The initial alignment methods on moving base can be commonly classified into three mainstream directions: transfer alignment, integrated alignment, and gyrocompass alignment. In transfer alignment, by means of velocity matching and attitude matching, a misaligned slave inertial navigation system can be aligned with the assistance of a master inertial navigation system. It can accomplish the initial alignment quickly and accurately, but the overall system is very complex [7]. Integrated alignment is an initial alignment method based on modern estimation theory and state space description. It can accomplish alignment rapidly and precisely by using modern filtering methods to estimate the misalignment angle. However, it is difficult to establish the absolutely accurate mathematical model and noise model of the system, and the large amount of calculation in alignment process always leads to poor instantaneity [8]. Gyrocompass alignment is built on the basis of classical control theory, so there is no need to establish accurate mathematical model and noise model. Its algorithm is simple, and the calculation amount is greatly reduced. However, as its fundamentals are established on static base or quasi-static base, when applied on moving base, gyrocompass alignment will be inaccurate or even impracticable with the effect of speed and acceleration.

In recent years, to improve the performance of initial alignment for SINS on moving base, gyrocompass alignment methods have already been analyzed by some researchers. 
In paper [9], azimuth axis rotating is used to improve the accuracy of compass loop, but the paper does not expand it to moving base [9]. Based on the principle of strap-down gyrocompass alignment, Cheng Xianghong from Southeastern University points out that the carrier velocity can affect the gyrocompass alignment and puts forward a calibration method for the inertial sensors of the SINS in the process of alignment on moving base [10]. Yan Gongmin from Northwestern Polytechnical University proposes a calculate method applied to the strap-down gyrocompass alignment on moving base [11]. As gyrocompass alignment needs less calculation amount but still remains reliable, it has a great application prospect for marine SINS alignment. Hwang et al. conduct precalibration using dual-electric compasses to minimize the error of spreader pose control [12].

However, few papers analyzed the problems faced by the gyrocompass alignment on moving base in a systemic and comprehensive way. Aiming at this problem, after analyzing the principle of classical gyrocompass initial alignment, this paper put forward a gyrocompass alignment method for SINS on moving base aided with Doppler velocity log (DVL) and deduces the process of algorithm realization in detail. Based on the characteristics of DVL's measuring error, we analyze the influence of velocity error of gyrocompass alignment on moving base and then establish a misalignment angle model. It can be found from the analysis that the most severe interference comes from the acceleration. The system parameters can be redesigned to restrain this kind of error, but it can also cause time growth. To shorten the gyrocompass initial alignment time, a data repeated calculation algorithm is also introduced [13].

The paper is organized as follows. In Section 2, the principle and realization of classical gyrocompass alignment for SINS on static base are introduced and the system characteristic is analyzed and in the end it leads to gyrocompass alignment on moving base. In Section 3, a DVL aided compass alignment method on moving base is proposed. The effect of velocity error, latitude error, and acceleration interference are analyzed, respectively, and the system parameters are redesigned to reduce the most serious effect. In Section 4, a fast compass alignment method based on reversed navigation algorithm is put forward, and the detailed calculating equations are given. In Section 5, simulations about the alignment methods mentioned above are done. In Section 6, a lake test with a certain type of SINS is carried out. Finally, conclusions are drawn in Section 7.

\section{Gyrocompass Alignment for SINS on Static Base}

2.1. Gyrocompass Alignment Principle. Gyrocompass alignment is commonly used in many kinds of inertial navigation systems [13-15] based on compass effect principle. This azimuth alignment method is proceeded after horizontal leveling adjust in application. By using control theory and adding dampings, it can make the platform coordinate approach the navigation coordinate gradually. In this section, the operating principle and implementation of gyrocompass

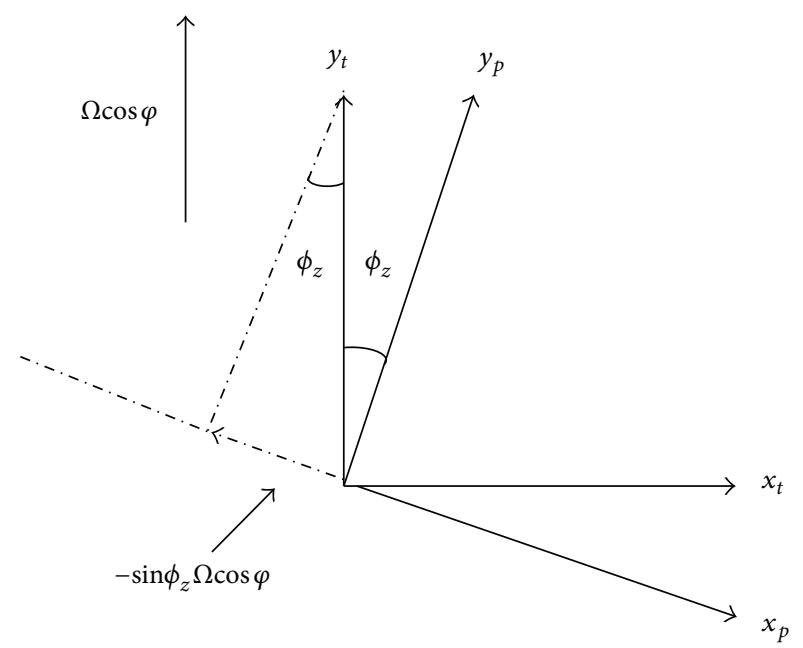

FIGURE 1: Schematic diagram of gyrocompass effect.

alignment for SINS on static base are described, and the accuracy on static base is also analyzed.

Compared with eastern horizontal loop, northern horizontal loop has an extra coupling term $\phi_{z} \omega_{i e} \cos \varphi$, which is in proportional relationship with the azimuth error angle. This term has the same function with eastern gyro drift, and it is an angular rate that comes from projection of the earth rotation angular rate in essence [16]. When there is an azimuth error angle $\phi_{z}$ between the platform coordinate system and the geographic coordinate system, the northern earth rotation angular rate $\Omega \cos \varphi$ will partly be projected to the platform coordinate system in eastern axis, and its projection value is $-\sin \phi_{z} \omega_{i e} \cos \varphi$. After coarse alignment, the projection value can approximately be simplified to $-\phi_{z} \omega_{i e} \cos \varphi$. Then the azimuth error angle can be coupled to northern horizontal loop by term $-\phi_{z} \omega_{i e} \cos \varphi$. This coupling relationship is defined as gyrocompass effect, as shown in Figure 1 [17].

Due to gyrocompass effect, the horizontal error angle $\phi_{x}$ is influenced additionally by the effect of azimuth error angle $\phi_{z}$, and the projection value of gravity acceleration along northern axis in platform coordinate system will change. It will lead to the change of velocity error in the northern loop. Making use of this coupling relationship, the gyrocompass alignment method controls the up axis gyro with the velocity error information and forms a new closed loop circuits called the gyrocompass loop [18]. Reasonable designed gyrocompass circuit parameters can make the system stable, fast, and more accurate; thus the gyrocompass alignment process can be accomplished.

\subsection{Realization of Gyrocompass Alignment for SINS on Static Base}

2.2.1. System Realization. The direction cosine matrix $C_{b}^{p}$ from carrier coordinate system to the platform coordinate system is an important matrix in the process of calculation. The angular velocity and acceleration information measured by IMU must be transformed into platform coordinate 


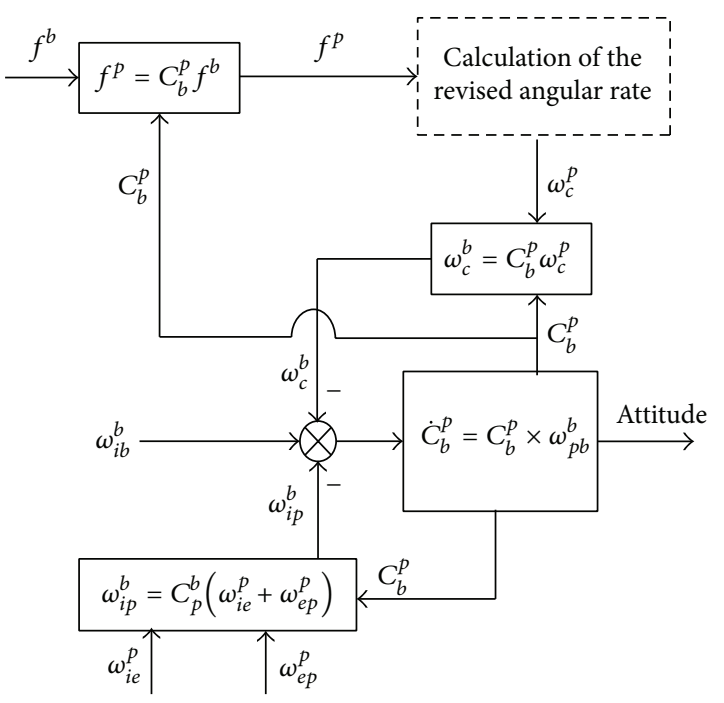

FIGURE 2: Schematic diagram of gyrocompass alignment of SINS.

system via matrix $C_{b}^{p}$ before participating in the navigation process. While $C_{b}^{p}$ has the same function with the physical platform of SINS, it is also called the mathematical platform.

As the mathematical platform is used instead of the physical platform in SINS, $C_{b}^{p}$ has become the control object of the revised angular velocity in gyrocompass method principle. The updating algorithm of the mathematical platform $C_{b}^{p}$ is as follows:

$$
\begin{gathered}
\omega_{p b}^{b}=\omega_{i b}^{b}-C_{p}^{b}\left(\omega_{e p}^{p}+\omega_{i e}^{p}\right), \\
\dot{C}_{b}^{p}=C_{b}^{p} \times \omega_{p b}^{b} .
\end{gathered}
$$

The angular velocity to control mathematical platform $C_{b}^{p}$ is $\omega_{p b}^{b}$. Considering the drift error of gyro and the error caused by interference movement of carrier, the revised angular velocity of SINS is added. After adding the control angular velocity $\omega_{c}^{p}$, the corresponding mathematical platform of SINS control equation is as follows:

$$
\omega_{p b}^{b}=\omega_{i b}^{b}-C_{p}^{b}\left(\omega_{e p}^{p}+\omega_{i e}^{p}\right)-C_{p}^{b}\left(\omega_{c}^{p}\right)
$$

The schematic diagram of gyrocompass initial alignment of SINS is given in Figure 2.

2.2.2. Calculation of the Revised Angular Rate. The revised angular rate can be obtained as shown in Figure 2. Figures 3 and 4 are the north channel and azimuth channel, respectively, in the compass alignment loop.

In Figure 3, as the dash line shows, $K_{1}$ is a damping term used to decrease the oscillation amplitude of Schuler loop; as the dash-dot line shows, $K_{2}$ is applied to shorten the system's natural period of oscillating period by $\sqrt{1+K_{2}}$ times. After term $\omega_{c x}^{p}$ there is a horizontal angle error caused by gyro drift and azimuth misalignment angle. As the double dash-dot line shows, $K_{3}$ is an energy storage term introduced to offset this error. All the $K$ values above can be calculated by damped coefficient $\xi$ and time constant $\sigma$ :

$$
\begin{aligned}
K_{1}=3 \sigma, \quad K_{2} & =\left(2+\frac{1}{\xi^{2}}\right) \frac{\sigma^{2}}{\omega_{s}^{2}}-1, \quad K_{3}=\frac{\sigma^{3}}{\xi^{2} \omega_{s}^{2}}, \\
\omega_{s} & =\sqrt{\frac{R}{g}}, \quad \sigma=\xi \omega_{n} .
\end{aligned}
$$

Compared with Figure $3, K_{3}$ is replaced by $K(s)$ in Figure 4 to reflect the compass effect term, $K(s)=$ $K_{3} / R \omega_{i e} \cos \varphi \cdot\left(s+K_{4}\right)$ and its purpose is to reduce the azimuth angle $\phi_{z}$ to an allowed range. All the $K$ values above can also be calculated by damped coefficient $\xi$ and time constant $\sigma$ :

$$
\begin{gathered}
K_{1}=K_{3}=2 \sigma, \quad K_{2}=\frac{\sigma^{2}+\omega_{n}^{2}}{\xi^{2} \cdot \omega_{s}^{2}}-1, \quad K_{4}=\frac{4 \sigma^{4}}{\xi^{2} \cdot \omega_{s}^{2}}, \\
\omega_{s}=\sqrt{\frac{R}{g}} .
\end{gathered}
$$

The alignment accuracy on static base is mainly decided by eastern and northern accelerometer zero bias $\nabla_{E}, \nabla_{N}$ and eastern gyro drift $\varepsilon_{E}$ :

$$
\begin{gathered}
\phi_{s x}=-\frac{1}{g} \nabla_{N}, \\
\phi_{s y}=\frac{1}{g} \nabla_{E}, \\
\phi_{s z}=\frac{\varepsilon_{E}}{\omega_{i e} \cos \varphi}+\frac{K_{4}\left(1+K_{2}\right) \varepsilon_{u}}{R \cdot K_{3}} .
\end{gathered}
$$

2.3. Static Base Gyrocompass Circuit Characteristic Analysis. Gyrocompass alignment on static base or quasi-static base has the following characteristics.

2.3.1. No External Acceleration Effect. Gyrocompass alignment changes the strap-down inertial navigation control system into a stable system in principle. However, Schuler loop of the system is destroyed and external acceleration impact is introduced into the system. According to Figures 3 and 4, although both of the two gyrocompass alignment horizontal loops will be infected by acceleration, the influence brought by motion acceleration can be ignored as the carrier accelerations $A_{N}$ and $A_{E}$ can be approximately regarded in this status.

2.3.2. Dispense with Updating of $\omega_{e p}^{p}$ and $\omega_{i e}^{p}$. According to Figure 2, besides the measured value of gyro and accelerator, there are inputs $\omega_{e p}^{p}$ and $\omega_{i e}^{p}$ in gyrocompass alignment realization process. In gyrocompass alignment system, there is only attitude calculation but no velocity and position calculation, so the value of $\omega_{e p}^{p}$ and $\omega_{i e}^{p}$ cannot be got except for bringing in external information. As the velocity of carrier is zero and the position of carrier remains the same on static base or quasi-static base, the value of $\omega_{e p}^{p}$ and $\omega_{i e}^{p}$ can be got directly without updating calculation. 


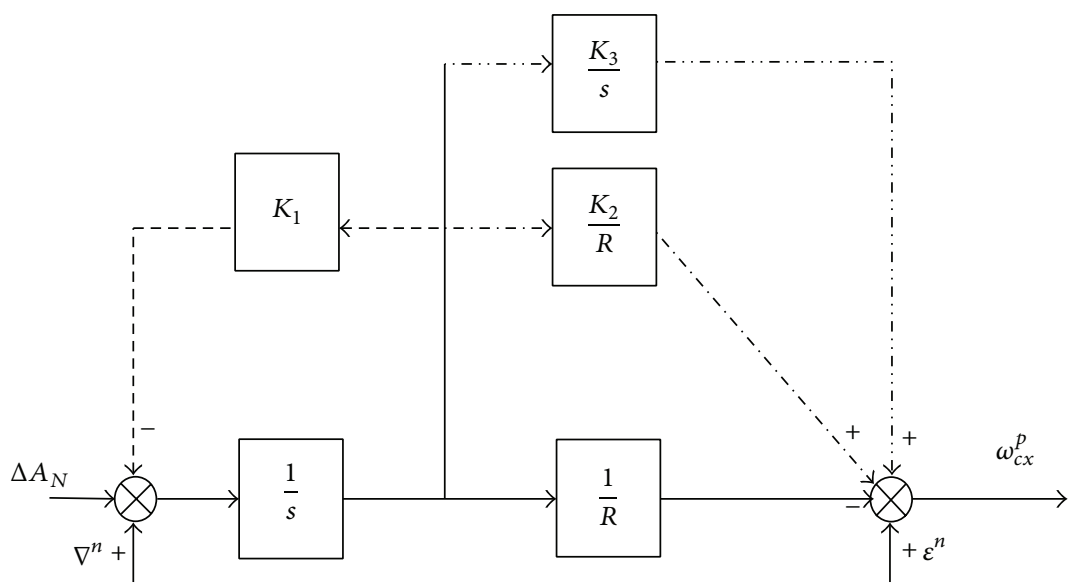

FIGURE 3: Schematic diagram of gyrocompass alignment in north channel.

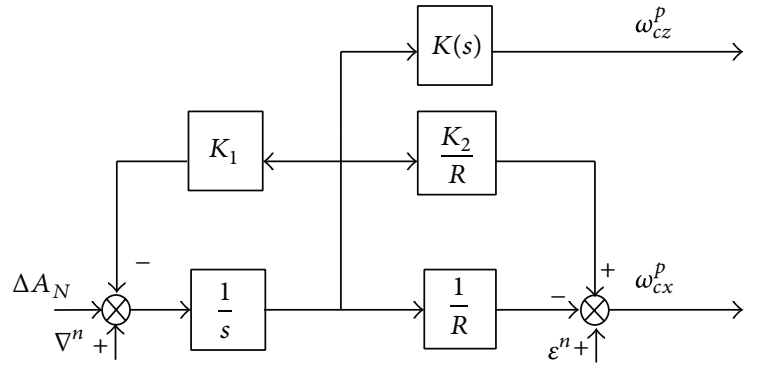

FIGURE 4: Schematic diagram of gyrocompass alignment in azimuth channel.

2.3.3. Fixed Instrument Error in Geography Coordinate System. SINS is strapped to carrier coordinate system, so its instrument error is defined in carrier coordinate system. Because the inertial navigation error equation is established in geography coordinate system, the analysis of instrument error has to be projected in geography coordinate system. As the carrier coordinate system remains relatively unchangeable with the geography coordinate system on static base, the instrument error in geography coordinate system is still constant.

2.4. Gyrocompass Alignment on Moving Base. Motion of carrier will change the relative position inevitably between geographical coordinate and inertial space. One reason is that the earth's rotation angular velocity $\omega_{i e}$ will change the direction of the earth coordinate system in inertial space; the other is that the movement of carrier on surface of the earth will cause relative rotation between geographical coordinate system and earth coordinate. Assuming the velocity of carrier is $V$ and the azimuth angle is $\psi$ in carrier coordinate, then their projections along north and east of geographical coordinate are $V_{N}=V \cdot \cos \psi$ and $V_{E}=V \cdot \sin \psi$, respectively.

As shown in Figure 5, the rotational angular velocity $\omega_{i n}^{n}$ of the geographical coordinate system relative to the inertial space can be regarded as sum of the earth's rotational angular velocity $\omega_{i e}$ and the relative rotational angular velocity $\omega_{e n}^{n}$

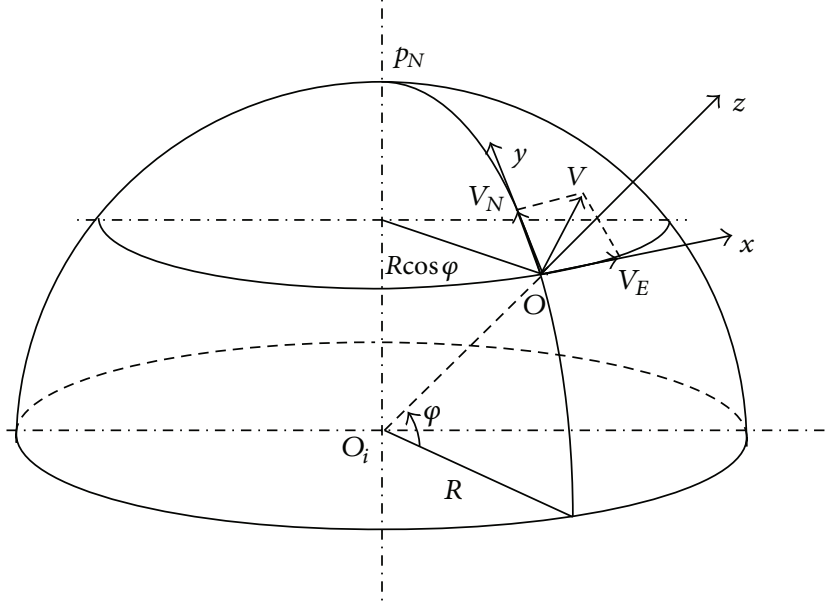

FIGURE 5: The projection in north and east of geographical coordinate.

between the geographical coordinate and the earth coordinate:

$$
\left[\begin{array}{l}
\omega_{x} \\
\omega_{y} \\
\omega_{z}
\end{array}\right]=\left[\begin{array}{c}
0 \\
\omega_{i e} \cos \varphi \\
\omega_{i e} \sin \varphi
\end{array}\right]+\left[\begin{array}{c}
-\frac{V \cos \psi}{R} \\
\frac{V \sin \psi}{R} \\
V \sin \psi \cdot \frac{\tan \varphi}{R}
\end{array}\right] .
$$

The output of gyroscope projected in navigation coordinates is $\omega_{i b}^{n}=\omega_{i e}^{n}+\varepsilon^{n}$ on static base, but on moving base it becomes $\omega_{i b}^{n}=\omega_{i e}^{n}+\omega_{e n}^{n}+\omega_{n b}^{n}+\varepsilon^{n}$. $\omega_{n b}^{n}$ can be regarded as zero in uniform motion. The output of accelerator projected in navigation coordinates is the gravitational acceleration $f^{n}=$ $-g^{n}+\nabla^{n}$ on static base. However it becomes $f^{n}=\left(2 \omega_{i e}^{n}+\right.$ $\left.\omega_{e n}^{n}\right) \times V^{n}-g^{n}+\nabla^{n}$ in uniform motion [13].

To make error analysis of misalignment caused by movement directly is relatively difficult. Therefore, the angular motion and the linear motion caused by movement are equivalent to gyro drift $\varepsilon_{d}^{n}$ and zero bias of acceleration $\nabla_{d}^{n}$ 


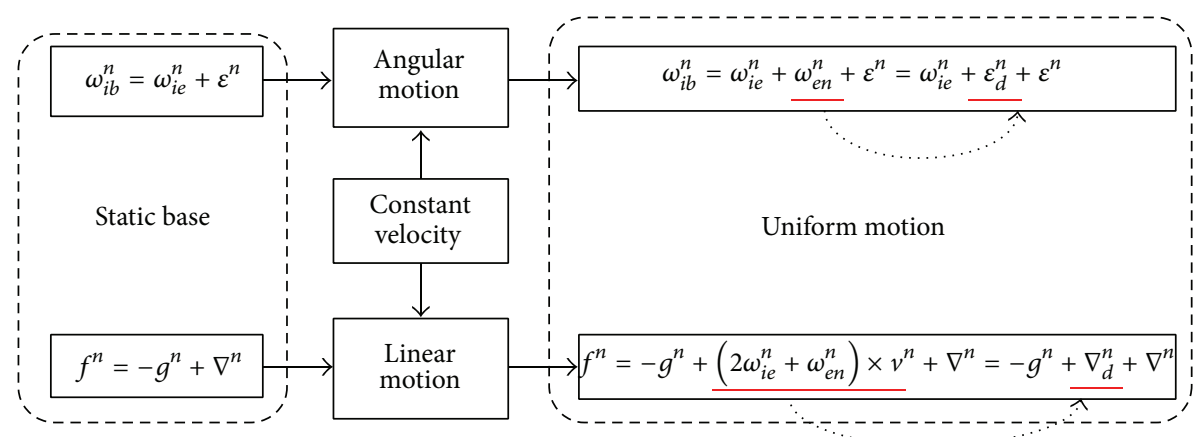

FIgURE 6: The equivalent error caused by uniform motion.

on static base correspondingly [19]. The equivalent error is shown in Figure 6.

The equivalent error can be calculated as follows:

$$
\begin{aligned}
\varepsilon_{d}^{n} & =\left[\begin{array}{lll}
\varepsilon_{d e} & \varepsilon_{d n} & \varepsilon_{d u}
\end{array}\right]^{T}=\omega_{e n}^{n} \\
& =\left[\begin{array}{lll}
-\frac{V \cos \psi}{R} & \frac{V \sin \psi}{R} & \frac{V \sin \psi \tan \varphi}{R}
\end{array}\right]^{T}, \\
\nabla_{d}^{n} & =\left[\begin{array}{l}
\nabla_{d e} \\
\nabla_{d n} \\
\nabla_{d n}
\end{array}\right]=\left(2 \omega_{i e}^{n}+\omega_{e n}^{n}\right) \times V^{n} \\
& =\left[\begin{array}{c}
-2 \omega_{i e} \cos \psi \sin \varphi \cdot V-\frac{V^{2} \sin \psi \cos \psi \tan \varphi}{R} \\
R \\
-\frac{V^{2} \sin \psi \cos \psi}{R}+2 \omega_{i e} \cos \varphi \cos \psi \cdot V+\frac{V^{2} \sin ^{2} \psi}{R}
\end{array}\right] .
\end{aligned}
$$

The final accuracy of misalignment angle along eastern, northern, and up orientation directions can be got by adding the instrument error and errors caused by carrier's motion into (5) to (7) that can be expressed as follows:

$$
\begin{gathered}
\phi_{s x}=-\frac{1}{g}\left(\nabla_{N}+\nabla_{d n}\right), \\
\phi_{s y}=\frac{1}{g}\left(\nabla_{E}+\nabla_{d e}\right), \\
\phi_{s z}=\frac{\varepsilon_{E}+\varepsilon_{d e}}{\omega_{i e} \cos \varphi}+\frac{K_{4}\left(1+K_{2}\right)\left(\varepsilon_{u}+\varepsilon_{d u}\right)}{R \cdot K_{3}} .
\end{gathered}
$$

In (12), $\omega_{i e}$ is earth's rotational velocity, $\varphi$ is latitude of carrier's position, and $\nabla_{E}, \nabla_{N}, \varepsilon_{E}$, and $\varepsilon_{U}$ are the equivalent gyro drift and equivalent accelerator bias in navigation coordinate system. The corresponding solution in motion will be introduced in the following sections.

\section{DVL Aided Gyrocompass Alignment on Moving Base}

3.1. DVL Aided Gyrocompass Alignment. The analysis in Section 2 gives conclusion that the influence factors of gyrocompass alignment become more complicated when the complexity of motion rises. From the perspective of system, the influencing form of acceleration in motion is similar to accelerometer bias, but its input value is much larger than accelerometer bias. What is more, from the perspective of DVL aided velocity information, error becomes more instable in motion.

From the analysis of (9), compared with gyrocompass alignment on static base, error compensations are needed in four parts, respectively, on moving base. They are angular velocity $\omega_{e p}^{p}$, earth rotation angular velocity $\omega_{i e}^{p}$, harmful acceleration $B^{p}$, and motion acceleration caused by sea waves.

The value of acceleration is only affected by waves in uniform motion, so it can be treated as disturbance. The other three parts can be calculated by the following equations:

$$
\begin{gathered}
\omega_{i e}^{p}=\left[\begin{array}{lll}
0 & \omega_{i e} \cos \varphi & \omega_{i e} \sin \varphi
\end{array}\right]^{T}, \\
\omega_{e p}^{p}=\left[\begin{array}{lll}
-\frac{V_{N}}{R} & \frac{V_{E}}{R} & \frac{V_{E}}{R \cos \varphi}
\end{array}\right]^{T}, \\
B^{p}=\left(\begin{array}{ll}
\omega_{e p}^{p}+2 \omega_{i e}^{p}
\end{array}\right) \times V^{p} .
\end{gathered}
$$

It can be found that the precise information of carrier's velocity and position is needed in compensation calculation. As the information cannot be obtained in gyrocompass alignment process, external information is essential to complete the calculation. If the initial position is known, with the assistance of DVL velocity information, the dynamic compensation can be calculated by the following methods after coarse alignment.

3.1.1. Velocity Projection Calculation. The velocity measured by DVL is $V^{b}$ in carrier coordinate system, but the velocity in navigation coordinate system has to be calculated. After coarse alignment, mathematical platform has been established and misalignment angle is controlled within a certain range, so the velocity in platform coordinate system can be got by projection calculation of mathematical platform. Set DVL measurement velocity as $V_{\mathrm{dvl}}^{b}$, and its expression is

$$
V_{\mathrm{dvl}}^{p}=C_{b}^{p} V_{\mathrm{dvl}}^{b}
$$

3.1.2. Latitude Calculation. After coarse alignment, the misalignment angle is controlled within a smaller range, so 


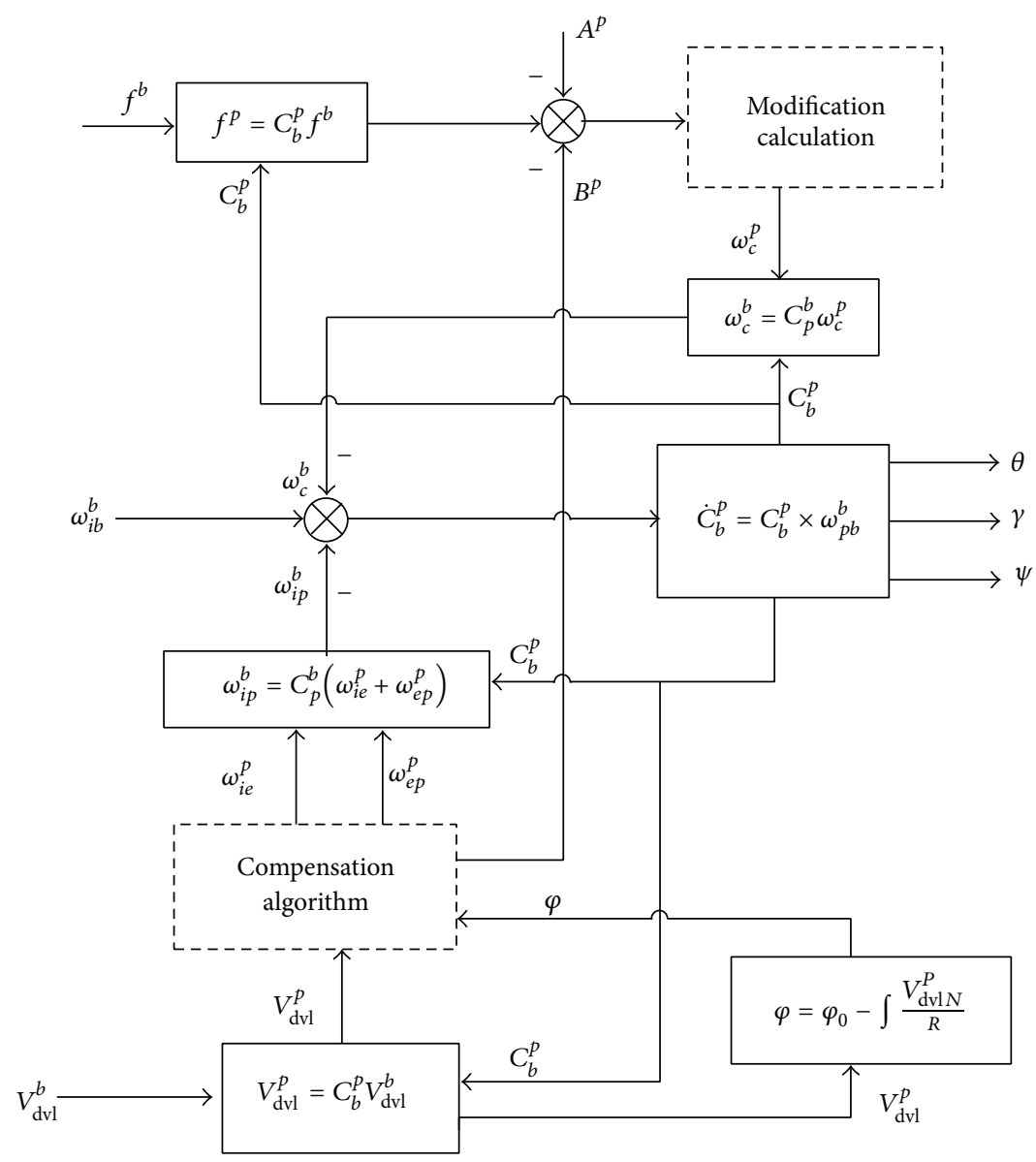

FIGURE 7: DVL aided gyrocompass alignment on moving base.

the carrier position can be got by integral calculation of the DVL velocity projection value $V_{\mathrm{dvl}}^{p}$ :

$$
\varphi=\varphi_{0}-\int \frac{V_{\mathrm{dvlN}}^{p}}{R} .
$$

$V_{\mathrm{dvlN}}^{p}$ is the projection of carrier velocity $V_{\mathrm{dvl}}^{p}$ along north in platform coordinate system.

3.1.3. Compensation Value Calculation. We can use latitude and velocity information to calculate compensation value $\omega_{i e}^{p}, \omega_{e p}^{p}$, and $B^{p}$ in (13)-(15). The implementation principle scheme of gyrocompass alignment on moving base is shown in Figure 7.

\subsection{Error Analysis of DVL Aided Compass Alignment on} Moving Base. There are still some error factors existing in the compensation calculation method mentioned in Section 3.1. On one hand, as there are errors in compensation calculation process, the calculation of DVL velocity $V_{\mathrm{dvl}}^{p}$ and latitude $\varphi$ will be effected accordingly. On the other hand, the error caused by sea waves is regarded as interference and ignored in compensation calculation. So we need to analyze the error of gyrocompass alignment from three aspects: velocity error, latitude error, and acceleration error.
3.2.1. The Effect of Velocity Error. As $V_{\text {dvl }}^{p}$ can be calculated by (16), error factors mainly come from the error of attitude matrix $C_{b}^{p}$ and the error of DVL measured velocity $V_{\mathrm{dvl}}^{b}$. In alignment process, misalignment angle becomes smaller gradually, so it is unnecessary to make further analysis of its influence.

The velocity $V_{\mathrm{dvl}}^{b}$ measured by DVL with constant error can be written as

$$
V_{\mathrm{dvl}}^{b}=V^{b}+\Delta V_{c}^{b}
$$

Equation (18) can be converted to the platform coordinate:

$$
V_{\mathrm{dvl}}^{p}=V^{p}+C_{b}^{p} \Delta V_{c}^{b}
$$

Due to swing of carrier and convergence of misalignment angle, there are some tiny variations in $C_{b}^{p}$. The swing with small amplitude can make $C_{b}^{p} \Delta V_{c}^{b}$ shake around a constant in limited range. So the error of $V_{\mathrm{dvl}}^{p}$ can be regarded as the sum of a constant error and a small high frequency oscillation. The calculation related to velocity is the angular velocity $\omega_{e p}^{p}$ and the harmful acceleration $B^{p}$. 
(1) The Influence $\omega_{e p}^{p}$ Calculation Errors. As shown in (14), velocity is linear to the angular rate, so the error in $\omega_{e p}^{p}$ by the effect of speed error $\delta V_{E}$ and $\delta V_{N}$ can be expressed as

$$
\delta \omega_{e p}^{p v}=\left[\begin{array}{lll}
-\frac{\delta V_{N}}{R} & \frac{\delta V_{E}}{R} & \frac{\delta V_{E}}{R \cos \varphi}
\end{array}\right]^{T} .
$$

Under the influence of this error, (2) can be rewritten as (21) on moving base:

$$
\begin{aligned}
\omega_{p b}^{\prime b} & =\omega_{i b}^{b}-C_{p}^{b}\left(\omega_{e p}^{p}+\delta \omega_{e p}^{p}+\omega_{i e}^{p}+\delta \omega_{i e}^{p}\right)-C_{p}^{b}\left(\omega_{c}^{p}\right) \\
& =\omega_{i b}^{b}-C_{p}^{b}\left(\delta \omega_{e p}^{p v}\right)-C_{p}^{b}\left(\omega_{e p}^{p}+\omega_{i e}^{p}\right)-C_{p}^{b}\left(\omega_{c}^{p}\right) .
\end{aligned}
$$

After adding gyroscopic drift, (21) can be written as

$$
\begin{aligned}
\omega_{p b}^{\prime b} & =\omega_{i b}^{b}+\varepsilon_{b}-C_{p}^{b}\left(\omega_{e p}^{p}+\omega_{i e}^{p}\right)-C_{p}^{b}\left(\omega_{c}^{p}\right) \\
& =\omega_{i b}^{b}+C_{p}^{b} \varepsilon_{p}-C_{p}^{b}\left(\omega_{e p}^{p}+\omega_{i e}^{p}\right)-C_{p}^{b}\left(\omega_{c}^{p}\right) .
\end{aligned}
$$

The comparison of (21) and (22) gives conclusion that the error of $\omega_{e p}^{p}$ and gyro drift in carrier coordinate system have the same influence form to attitude updating calculation. If it is considered as equivalent gyro drift $\varepsilon_{V d e}$ and $\varepsilon_{V d u}$, the error with small high-frequency oscillation will be restrained by the system and constant error is the only thing to be considered. Based on (7), with the effect of constant error, the alignment error caused by equivalent gyro drift in form (20) is as follows:

$$
\begin{aligned}
\phi_{z}^{\delta V_{1}} & =\frac{\varepsilon_{V d e}}{\omega_{i e}^{n}}+\frac{K_{z}\left(1+K_{2}\right) \varepsilon_{V d u}}{R \cdot K_{3}} \\
& =\frac{1}{\omega_{i e} \cos \varphi} \cdot\left(-\frac{\delta V_{N}}{R}\right)+\frac{K_{z}\left(1+K_{2}\right)}{K_{3}} \cdot \frac{\delta V_{E}}{R^{2} \cos \varphi} .
\end{aligned}
$$

As analyzed above, east gyro drift $-\delta V_{N} / R$ has greater influence on azimuth angle than azimuth gyro drift $\delta V_{E} / R \cos \varphi$, so the second part in (23) can be neglected and (23) can be simplified as

$$
\phi_{z}^{\delta V_{1}}=\frac{1}{\omega_{i e} \cos \varphi} \cdot\left(-\frac{\delta V_{N}}{R}\right) .
$$

(2) The Influence of Harmful Acceleration Error. As shown in Figure 7, the influence form of harmful acceleration $B^{p}$ is similar to $A^{p}$. Therefore the influence of harmful acceleration and the accelerometer bias can be written in the same form, and the acceleration bias error analysis method can also be used to analyze the influence of harmful acceleration.

The projection of errors caused by harmful acceleration along east and north of the platform is as follows:

$$
\begin{gathered}
B_{E}^{p}=\left(2 \omega_{i e} \sin \varphi+\frac{V_{E}}{R \cos \varphi}\right) \cdot V_{N}, \\
B_{N}^{p}=-\left(2 \omega_{i e} \sin \varphi+\frac{V_{E}}{R \cos \varphi}\right) \cdot V_{E} .
\end{gathered}
$$

With the effect of velocity errors $\delta V_{E}$ and $\delta V_{N}$, (25) can be converted as

$$
\begin{aligned}
& B_{E}^{\prime p}=\left(2 \omega_{i e} \sin \varphi+\frac{V_{E}+\delta V_{E}}{R \cos \varphi}\right) \cdot\left(V_{N}+\delta V_{N}\right), \\
& B_{N}^{\prime p}=-\left(2 \omega_{i e} \sin \varphi+\frac{V_{E}+\delta V_{E}}{R \cos \varphi}\right) \cdot\left(V_{E}+\delta V_{E}\right) .
\end{aligned}
$$

The approximate value of harmful acceleration error can be got by subtracting (26) from (25), and the result is

$$
\begin{gathered}
\delta B_{E}^{p}=2 \omega_{i e} \sin \varphi \cdot \delta V_{N}+\frac{\delta V_{N} \cdot V_{E}+\delta V_{E} \cdot V_{N}+\delta V_{E} \cdot \delta V_{N}}{R \cos \varphi}, \\
\delta B_{N}^{p}=-\left(2 \omega_{i e} \sin \varphi \cdot \delta V_{E}+\frac{2 \delta V_{E} \cdot V_{E}+\delta V_{E}^{2}}{R \cos \varphi}\right) .
\end{gathered}
$$

There is little change in latitude, so $\varphi$ can be considered as a constant in alignment process. The velocity and its error can be considered as sum of constant and high frequency oscillation. As the swing frequency is high, the system has an inhibition to this oscillation error, so its influence is relatively weak, so (26) can be analyzed as the constant gyro drift. The harmful acceleration errors $\delta B_{E}^{p}$ and $\delta B_{N}^{p}$ and the constant accelerometer bias $\nabla_{B d e}, \nabla_{B d n}$ are equivalent, so according to (6) and (7), error angle can be obtained in (27) with the influence of the equivalent accelerometer bias:

$$
\begin{aligned}
\phi_{x}^{\delta V_{2}}= & -\frac{\nabla_{B d n}}{g} \\
=-\frac{1}{g} \cdot( & -2 \omega_{i e} \sin \varphi \cdot \delta V_{N} \\
& \left.-\frac{\delta V_{N} \cdot V_{E}+\delta V_{E} \cdot V_{N}+\delta V_{E} \cdot \delta V_{N}}{R \cos \varphi}\right), \\
\phi_{y}^{\delta V_{2}}=\frac{\nabla_{B d e}}{g}=\frac{1}{g} \cdot( & 2 \omega_{i e} \sin \varphi \cdot \delta V_{E} \\
& \left.+\frac{2 \delta V_{E} \cdot V_{E}+\delta V_{E}^{2}}{R \cos \varphi}\right) .
\end{aligned}
$$

Synthesizing the misalignment angles caused by two parts of the velocity error, the error equation can be rewritten as

$$
\begin{aligned}
& \phi_{x}^{\delta V}=\frac{1}{g} \cdot( 2 \omega_{i e} \sin \varphi \cdot \delta V_{N} \\
&+\left.\frac{\delta V_{N} \cdot V_{E}+\delta V_{E} \cdot V_{N}+\delta V_{E} \cdot \delta V_{N}}{R \cos \varphi}\right), \\
& \phi_{y}^{\delta V}=\frac{1}{g} \cdot(\left.2 \omega_{i e} \sin \varphi \cdot \delta V_{E}+\frac{2 \delta V_{E} \cdot V_{E}+\delta V_{E}^{2}}{R \cos \varphi}\right), \\
& \phi_{z}^{\delta V}=\frac{1}{\omega_{i e} \cos \varphi} \cdot\left(-\frac{\delta V_{N}}{R}\right) .
\end{aligned}
$$

3.2.2. The Effect of Latitude Error. Latitude calculation can be mainly divided into two parts: one is the calculation of $\omega_{i e}^{p}$ 
and $\omega_{e p}^{p}$ and the other is the calculation of the parameters in feedback loop. They are analyzed, respectively, in the following sections.

(1) The Influence Caused by Calculation Error of $\omega_{i e}^{p}$ and $\omega_{e p}^{p}$. From (13) and (14), we can know that the calculation of $\omega_{i e}^{p}$ and $\omega_{e p}^{p}$ is related to the latitude error. Set $\varphi^{\prime}$ as inaccurate latitude, and $\delta \varphi^{\prime}=\varphi^{\prime}-\varphi$ as latitude error.

While there exits error in latitude, the value of $\omega_{i e}^{p}$ can be calculated by

$$
\omega_{i e}^{\prime p}=\left[\begin{array}{lll}
0 & \omega_{i e} \cos \varphi^{\prime} & \omega_{i e} \sin \varphi^{\prime}
\end{array}\right]^{T} .
$$

Therefore, the miscalculation of $\omega_{i e}^{p}$ can be got by subtracting (13) from (30):

$$
\begin{aligned}
\delta \omega_{i e}^{p \varphi} & =\left[\begin{array}{c}
0 \\
\omega_{i e}\left(\cos \varphi^{\prime}-\cos \varphi\right) \\
\omega_{i e}\left(\sin \varphi^{\prime}-\sin \varphi\right)
\end{array}\right] \\
= & {\left[\begin{array}{c}
0 \\
\omega_{i e} \cdot\left(-2 \sin \left(\varphi+\frac{\delta \varphi}{2}\right) \sin \delta \varphi\right) \\
\omega_{i e} \cdot\left(2 \cos \left(\varphi+\frac{\delta \varphi}{2}\right) \sin \delta \varphi\right)
\end{array}\right] . }
\end{aligned}
$$

While there exists an error, the calculation value of $\omega_{e p}^{p}$ is

$$
\omega_{e p}^{\prime p}=\left[\begin{array}{lll}
-\frac{V_{N}}{R} & \frac{V_{E}}{R} & \frac{V_{E}}{R \cos \varphi^{\prime}}
\end{array}\right]^{T} .
$$

The calculation error of $\omega_{e p}^{p}$ can be got by subtracting (14) from (32):

$$
\begin{aligned}
& \delta \omega_{e p}^{p \varphi}=\left[\begin{array}{lll}
0 & 0 & \frac{V_{E}}{R \cos \varphi}-\frac{V_{E}}{R \cos \varphi^{\prime}}
\end{array}\right]^{T}
\end{aligned}
$$

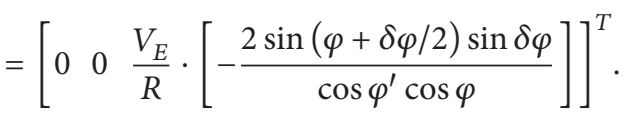

Similar to the analysis of $\delta \omega_{e p}^{p v}, \delta \omega_{e p}^{p \varphi}$ and $\delta \omega_{i e}^{p \varphi}$ can be treated as equivalent gyro drift; then combining with (31) and (33), the gyro drift error can be written as the sum of $\delta \omega_{e p}^{p \varphi}$ and $\delta \omega_{i e}^{p \varphi}:$

$$
\delta \omega_{e p}^{p}+\delta \omega_{i e}^{p}=\left[\begin{array}{c}
0 \\
\omega_{i e} \sin \delta \varphi \cdot\left(-2 \sin \left(\varphi+\frac{\delta \varphi}{2}\right)\right) \\
\omega_{i e} \sin \delta \varphi \cdot\left(2 \cos \left(\varphi+\frac{\delta \varphi}{2}\right)\right)-\frac{\sin \delta \varphi}{R} \cdot\left[\frac{2 V_{E} \sin (\varphi+\delta \varphi / 2)}{\cos \varphi^{\prime} \cos \varphi}\right]
\end{array}\right] .
$$

From the analysis of (23), as $\cos \varphi$ is in the denominator, the error will be infinite in theory when the carrier is sailing in high latitudes. However, the gyrocompass alignment is applied in mid or low latitudes, so this situation is out of consideration [20]. As the radius of the earth is very large, the carrier's change in position can only lead to tiny change in latitude, and the latitude error $\delta \varphi$ is even fainter. Dead reckoning latitude error terms $\omega_{i e} \sin \delta \varphi$ and $\sin \delta \varphi / R$ in (34) can be neglected compared with the relatively larger error of velocity.

(2) The Influence of Corrected Angular Velocity. The corrected angle rate of gyrocompass alignment needs to be calculated through the form of Figures 3 and 4 . Therefore, the values of parameters $K_{1}, K_{2}, K_{3}$, and $K_{4}$ are needed to be determined. Equation (4) in Section 2.2 shows that the azimuth loop parameters are calculated as follows:

$$
\begin{gathered}
K_{1}=K_{3}=2 \xi \omega_{n}, \\
K_{2}=\frac{R \omega_{n}^{2}\left(1+\xi^{2}\right)}{g}-1, \\
K(s)=\frac{R \xi^{2} \omega_{n}^{4}}{g} .
\end{gathered}
$$

$\xi$ and $\omega_{n}$ are adjustable variables in system; $\omega_{i e}$ and $g$ are known values. When errors occurred in $\varphi$, the accurate value of parameter $K(s)$ cannot be obtained, and the incorrect $K(s)$ will affect the convergence speed of the system. However, as the value of $\delta \varphi$ is small, this influence on the convergence speed is weak.

3.2.3. The Effect of Acceleration Interference. The main acceleration of carrier is caused by wind and waves in uniform straight line motion. Assuming that acceleration caused by waves is a sine periodic oscillation, and its value is $A_{p}=$ $A \sin (\omega t+\varphi)$, then the velocity error can be got by integrating the acceleration. It is a cosine periodic oscillation and its value is $V_{p}=(A / \omega) \cos \varphi-(A / \omega) \cos (\omega t+\varphi)$. For the acceleration interference, there is harmful acceleration interference caused by the velocity error except for $A^{p}$, and all the above factors can be equivalent to acceleration zero biases $\nabla_{A d n}$ and $\nabla_{A d e}$ as described in Section 3.2.1:

$\nabla_{A d}=\left[\begin{array}{c}\nabla_{\text {Ade }} \\ \nabla_{A d n} \\ \nabla_{A d u}\end{array}\right]=\left(2 \omega_{i e}^{n}+\omega_{P}^{n}\right) \times V_{P}^{n}$ 


$$
=\left[\begin{array}{c}
A_{N}+2 \omega_{i e} \sin \psi \sin \varphi \cdot V_{P}+\frac{V_{p}^{2} \sin ^{2} \psi \tan \varphi}{R} \\
A_{E}-2 \omega_{i e} \cos \psi \sin \varphi \cdot V_{P}-\frac{V_{p}^{2} \sin \psi \cos \psi \tan \varphi}{R} \\
\frac{-V_{p}^{2} \sin \psi \cos \psi}{R}+2 \omega_{i e} \cos \varphi \cos \psi \cdot V_{p}+\frac{V_{p}^{2} \sin ^{2} \psi}{R}
\end{array}\right] .
$$

There are also equivalent gyro drifts in three directions produced by velocity error: $\varepsilon_{A d n}, \varepsilon_{A d e}$, and $\varepsilon_{A d u}$ :

$$
\begin{aligned}
\varepsilon_{\text {Ad }} & =\left[\begin{array}{lll}
\varepsilon_{\text {Ade }} & \varepsilon_{\text {Adn }} & \varepsilon_{\text {Adu }}
\end{array}\right]^{T}=\omega_{p}^{n} \\
& =\left[\begin{array}{lll}
-\frac{V_{P} \cos \psi}{R} & \frac{V_{P} \sin \psi}{R} & \frac{V_{P} \sin \psi \tan \varphi}{R}
\end{array}\right]^{T} .
\end{aligned}
$$

The horizontal alignment is mainly affected by acceleration bias, and the azimuth alignment is mainly affected by east gyro drift. At this time, the misalignment angle equation in the frequency domain can be written as

$$
\begin{gathered}
\phi_{x}^{A}(s)=\frac{-\left(\left(1+2 \xi^{2}\right) \omega_{n}^{2} / g\right)\left(s+\xi \omega_{n} /\left(1+2 \xi^{2}\right)\right)}{\left(s^{2}+2 \xi \omega_{n} s+\omega_{n}^{2}\right)\left(s+\xi \omega_{n}\right)} \cdot \nabla_{A d n}(s), \\
\phi_{y}^{A}(s)=\frac{\left(\left(1+2 \xi^{2}\right) \omega_{n}^{2} / g\right)\left(s+\xi \omega_{n} /\left(1+2 \xi^{2}\right)\right)}{\left(s^{2}+2 \xi \omega_{n} s+\omega_{n}^{2}\right)\left(s+\xi \omega_{n}\right)} \cdot \nabla_{A d e}(s), \\
\phi_{z}^{A}(s)=\frac{\xi^{2} \omega_{n}^{4} / \Omega \cos \varphi}{\left(s^{2}+2 \xi \omega_{n} s+\omega_{n}^{2}\right)\left(s+\xi \omega_{n}\right)^{2}} \cdot \varepsilon_{A d n}(s) .
\end{gathered}
$$

The acceleration $A_{P}$ produced by waves is in form of periodic oscillation with small amplitude and high frequency. Its input frequency is generally limited in $\left[\omega_{n},+\infty\right)$, so the effect of $A_{P}$ on misalignment angle can be greatly reduced by lowering the value of $\omega_{n}$. The effect of uniform motion interference acceleration can be suppressed by changing the parameters in the system, but the alignment time will also be increased accordingly.

In this section the error of gyrocompass alignment in uniform straight line motion is analyzed from three aspects: latitude error, velocity error, and acceleration error. The influence of velocity error and latitude error on the misalignment angle is too weak to be considered, but the interference acceleration brought by swing and waves in motion has a great influence on the misalignment angles. We can reduce this influence by changing the system parameters, but the alignment time will be increased accordingly.

\section{A Rapid Implementation of DVL Aided Gyrocompass Method in Alignment}

In platform inertial navigation system (PINS), it is difficult for the platform to revert to former states and adding a new control method again, whereas for SINS, assuming that the storage capacity of navigation computer is large and computing power is strong enough, it is feasible for the navigation computer to make a storage of the sampling data of SINS and calculate the data repeatedly with different kinds of algorithms. By using this kind of repeated calculation method, the increased alignment time caused by changes in parameters can be solved to some extent.

There exists a certain convergence in the gyrocompass alignment process, and it is the main factor to affect the alignment time, so alignment time can be shortened by reducing the convergent time or accomplishing the convergence in other processes. By calculating the data repeatedly, with the convergence completed in this repeated calculation process, the original alignment process is shortened, which in turn reduces the alignment time although the overall convergence process did not change.

From the analysis above, the conception and structure of an improved rapid alignment algorithm is given as follows: the gyro and acceleration sampling data of SINS can be regarded as a group of time series. The traditional navigation process calculates this data series according to time order, and real-time navigation results can be got without the stored procedure. For the same reason, if this data series is stored by navigation computer, they can be calculated backward to conduct the processing and analyzing procedure as well. It is called data repeated calculation algorithm in this paper, and, by analyzing the sampling data forward and backward repeatedly, the accuracy is increased and the actual length of the analyzed data series is shortened, in return reduced the alignment time. The schematic diagram of the data repeated calculation algorithm is shown in Figure 8 , in which $\Delta T$ is the sampling period.

Attitude, velocity, and position calculation of the compass alignment algorithm for SINS are expressed in the following differential equation:

$$
\begin{gathered}
\dot{C}_{b}^{n}=C_{b}^{n} \Omega_{n b}^{n}, \\
\dot{v}^{n}=C_{b}^{n} f_{i b}^{b}-\left(2 \omega_{i e}^{n}+\omega_{e n}^{n}\right) \times v^{n}+g^{n}, \\
\dot{\varphi}=\frac{v_{N}^{n}}{R}, \quad \dot{\lambda}=\frac{v_{E}^{n} \sec \varphi}{R} .
\end{gathered}
$$

Among them

$$
\begin{gathered}
\Omega_{n b}^{b}=\left(\omega_{n b}^{b} \times\right), \quad \omega_{n b}^{b}=\omega_{i b}^{b}-\left(C_{b}^{n}\right)^{T}\left(\omega_{i e}^{n}+\omega_{e n}^{n}\right), \\
g^{n}=[0,0,-g]^{T}, \\
\omega_{i e}^{n}=\left[0, \omega_{i e} \cos \varphi, \omega_{i e} \sin \varphi\right]^{T}, \\
\omega_{e n}^{n}=\left[-\frac{v_{N}^{n}}{R}, \frac{v_{E}^{n}}{R}, \frac{v_{E}^{n} \tan \varphi}{R}\right]^{T} .
\end{gathered}
$$

$\delta v^{n}$ is obtained by compass circuit and $C_{b}^{n}, v^{n}=$ $\left[v_{E}^{n}, v_{N}^{n}, v_{U}^{n}\right]^{T}, \varphi$, and $\lambda$ are inertial attitude matrix, speed, latitude, and longitude, respectively. $\omega_{i b}^{b}$ and $f_{i b}^{b}$ are measuring gyro angular velocity and measuring acceleration, respectively. $\omega_{i e}$ and $g$ are the angle rate of the earth and the local acceleration of gravity, respectively. $R$ is the radius of earth. Operator $i(\bullet \times)$ is the antisymmetric matrix composed by - vector. Assuming the sampling period of gyroscope 


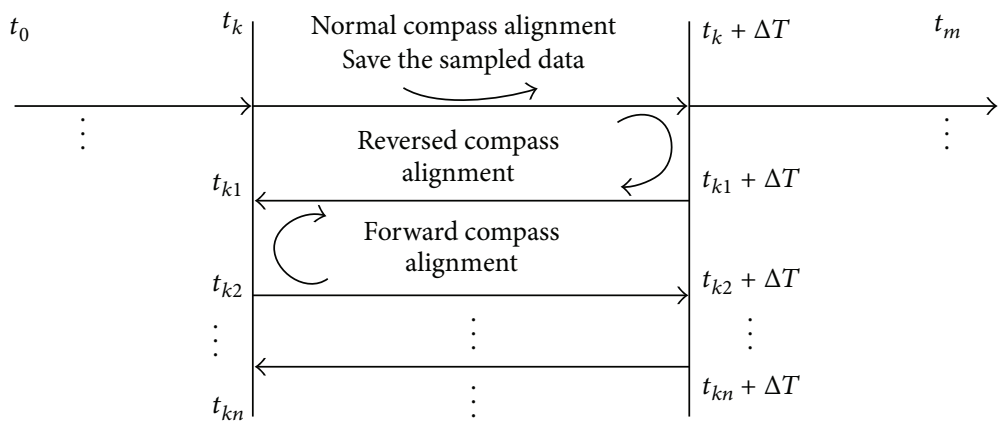

FIgURE 8: Data repeated calculation alignment process diagram.

and accelerometer in SINS are both $\Delta T$, the differential equations ((39a), (39b), and (39c)) are discrete recursion method suitable for computer calculating:

$$
\begin{gathered}
C_{b k}^{n}=C_{b k-1}^{n}\left(I+\Delta T \cdot \Omega_{n b k}^{b}\right) \\
v_{k}^{n}=v_{k-1}^{n}+\Delta T \cdot\left[C_{b k-1}^{n} f_{i b k}^{b}-\left(2 \omega_{i e k-1}^{n}+\omega_{e n k-1}^{n}\right) \times v_{k-1}^{n}+g^{n}\right] \\
\varphi_{k}=\varphi_{k-1}+\frac{\Delta T \cdot v_{N k-1}^{n}}{R} \\
\lambda_{k}=\lambda_{k-1}+\frac{\Delta T \cdot v_{E k-1}^{n} \sec \varphi_{k-1}}{R}
\end{gathered}
$$

Among them

$$
\begin{gathered}
\Omega_{n b k}^{b}=\left(\omega_{n b k}^{b} \times\right), \\
\omega_{n b k}^{b}=\omega_{i b k}^{b}-\left(C_{b k-1}^{n}\right)^{T}\left(\omega_{i e k-1}^{n}+\omega_{e n k-1}^{n}+\omega_{c k-1}^{n}\right), \\
\omega_{i e k}^{n}=\left[0, \omega_{i e} \cos \varphi, \omega_{i e} \sin \varphi_{k}\right]^{T}, \\
\omega_{e n k}^{n}=\left[-\frac{v_{N k}^{n}}{R}, \frac{v_{E k}^{n}}{R}, \frac{v_{E k}^{n} \tan \varphi_{k}}{R}\right]^{T} \quad(k=1,2,3, \ldots) .
\end{gathered}
$$

$\omega_{c}^{n}=\delta v^{n} / R, \delta v^{n}$ can be obtained by compass circuit.

From the equations above, if we take the opposite value of gyro output and the earth rotation angle rate of the forward navigation algorithm, set the initial value of the algorithm as $\widehat{C}_{b 0}^{n}=C_{b m}^{n}, \widehat{v}_{0}^{n}=-v_{m}^{n}, \widehat{\varphi}_{0}=\varphi_{m}$, and $\hat{\lambda}_{0}=\lambda_{m}$, and calculate the sampling data repeatedly, the repeated calculation algorithm can be achieved. It has the same expression with the forward navigation calculation, and the reversed navigation calculating process from $t_{m}$ (point B) to $t_{0}$ (point A) can simply be got by using this algorithm. Regardless of calculating error, attitude matrix and position coordinates are both equal at the same time of the data series while the velocity has the same absolute value with opposite sign in forward and reversed calculation.
The reversed navigation algorithm of SINS is as follows:

$$
\begin{aligned}
C_{b k-1}^{n}= & C_{b k}^{n}\left(I+\Delta T \cdot \Omega_{n b k}^{b}\right)^{-1} \\
\approx & C_{b k}^{n}\left(I-\Delta T \cdot \Omega_{n b k}^{b}\right) \approx C_{b k}^{n}\left(I+\Delta T \cdot \widetilde{\Omega}_{n b k-1}^{b}\right), \\
v_{k-1}^{n}= & v_{k}^{n}-\Delta T \\
& \cdot\left[C_{b k-1}^{n} f_{i b k}^{b}-\left(2 \omega_{i e k-1}^{n}+\omega_{e n k-1}^{n}\right) \times v_{k-1}^{n}+g^{n}\right] \\
\approx & v_{k}^{n}-\Delta T \cdot\left[C_{b k}^{n} f_{i b k-1}^{b}-\left(2 \omega_{i e k}^{n}+\omega_{e n k}^{n}\right) \times v_{k}^{n}+g^{n}\right], \\
\varphi_{k-1} & =\varphi_{k}-\frac{\Delta T \cdot v_{N k-1}^{n}}{R} \approx \varphi_{k}-\frac{\Delta T \cdot v_{N k}^{n}}{R}, \\
\lambda_{k-1}= & \lambda_{k}-\frac{\Delta T \cdot v_{E k-1}^{n} \sec \varphi_{k-1}}{R} \approx \lambda_{k}-\frac{\Delta T \cdot v_{E k}^{n} \sec \varphi_{k}}{R} .
\end{aligned}
$$

Among them,

$$
\begin{gathered}
\widetilde{\Omega}_{n b k-1}^{b}=\left(\widetilde{\omega}_{n b k-1}^{b} \times\right) \\
\widetilde{\omega}_{n b k-1}^{b}=-\left[\omega_{i b k-1}^{b}-\left(C_{b k}^{n}\right)^{T}\left(\omega_{i e k}^{n}+\omega_{e n k}^{n}+\omega_{c k}^{n}\right)\right] .
\end{gathered}
$$

\section{Simulation}

5.1. Simulation Experiment of Traditional Gyrocompass Alignment Method. The comparison of gyrocompass alignment on static base and moving base is given, respectively, as follows.

5.1.1. The Simulation Conditions. Simulation is proceeded at latitude $\varphi=45.7796^{\circ}$ and longitude $\lambda=126.6705^{\circ}$ (Harbin area); in order to make a better observation of effect in motion, the triaxial gyro drift of SINS is set as $0.01^{\circ} / \mathrm{h}$ and the bias of accelerator is set as $0.0001 \mathrm{~g}$. The parameters of 
TABLE 1: Misalignments of gyrocompass alignment in different conditions.

\begin{tabular}{lccc}
\hline & $\begin{array}{c}\text { Eastern error } \\
\text { angle }\left(^{\circ}\right)\end{array}$ & $\begin{array}{c}\text { Northern error } \\
\text { angle }\left(^{\circ}\right)\end{array}$ & $\begin{array}{c}\text { Azimuth error } \\
\text { angle }\left(^{\circ}\right)\end{array}$ \\
\hline In motion & $-4.16 \times 10^{-3}$ & $-4.34 \times 10^{-3}$ & 1.211 \\
Static base & $0.22 \times 10^{-3}$ & $0.21 \times 10^{-3}$ & -0.059 \\
\hline
\end{tabular}

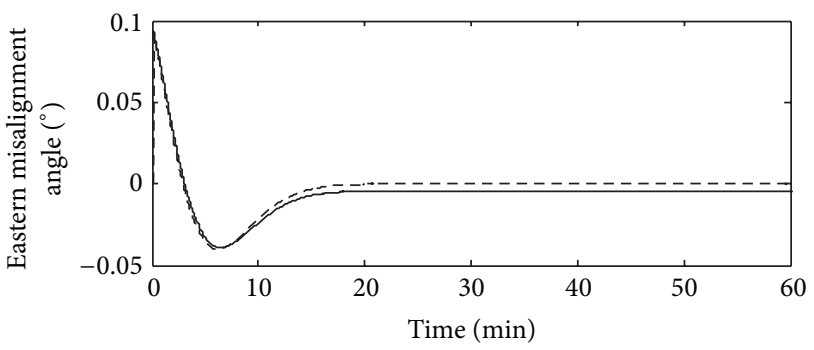

FIGURE 9: Comparison of the misalignment in east axis.

gyrocompass alignment are set as $\xi=0.707$ and $\omega_{n}=0.008$; it means that the alignment parameters configuration is

$$
\begin{gathered}
k_{1}=k_{2}=0.0113, \\
k_{E}=k_{N}=9.81 \times 10^{-6}, \\
k_{U}=4.1 \times 10^{-6} .
\end{gathered}
$$

Assuming that the carrier's speed is $10 \mathrm{~m} / \mathrm{s}$ and heading is along $315^{\circ}$, the swing and sway of sailing are set as sinusoidal oscillation form. The extent of pitch, roll, and yaw axis swing is set as $6^{\circ}, 8^{\circ}$, and $5^{\circ}$, and the periods are set as $8 \mathrm{~s}, 6 \mathrm{~s}$, and $10 \mathrm{~s}$; the extent of surge, sway, and heave is set as $0.1 \mathrm{~m} / \mathrm{s}^{2}$, and the periods are $5 \mathrm{~s}$. Set the axis misalignment angles of coarse alignment as $0.1^{\circ}, 0.1^{\circ}$, and $1^{\circ}$, respectively.

5.1.2. The Simulation Results. The gyrocompass alignment method is used both on static base and in uniform motion. After maintaining one hour of alignment process, the alignment results in both conditions are compared and shown in Figures 6-8. The thick dash line represents gyrocompass alignment on static base and the thin solid line represents gyrocompass alignment in uniform motion.

The error curves in Figures 9, 10, and 11 indicate that the gyrocompass alignment has good performance on static base, but there is constant error caused by velocity which exists during the alignment process while the ship is in motion. By choosing the mean value of alignment errors in two minutes before the alignment process ends, the results of both conditions are recorded in Table 1.

In theory, three misalignment angles on static base can be obtained by substituting the gyro drift and accelerator zero bias into (5) $-(7)$. The values are $0.209 \times 10^{-3}\left(^{\circ}\right), 0.223 \times$ $10^{-3}\left({ }^{\circ}\right)$, and $0.062\left(^{\circ}\right)$, respectively, which is nearly the same to the simulation results. Then a conclusion can be drawn that velocity will cause a sharp alignment error. Substitute velocity into (10)-(12); three misalignment angles can be obtained as

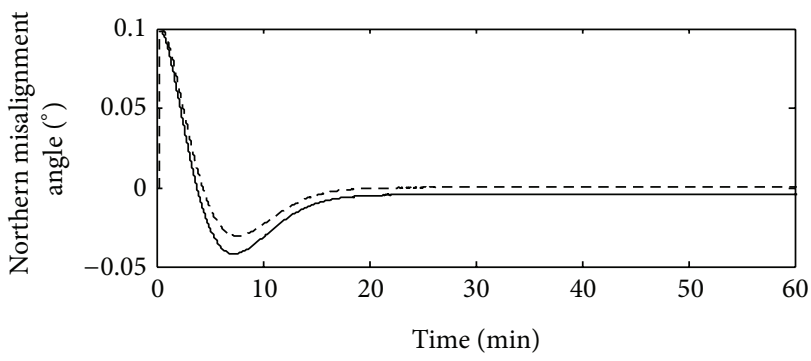

FIGURE 10: Comparison of the misalignment in north axis.

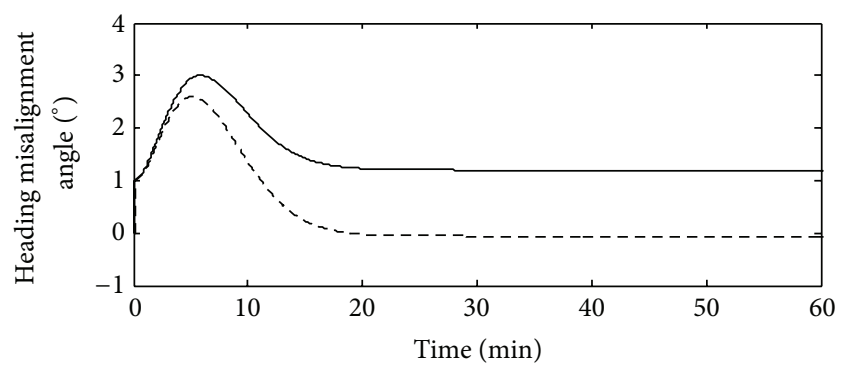

FIgURE 11: Comparison of the heading misalignment.
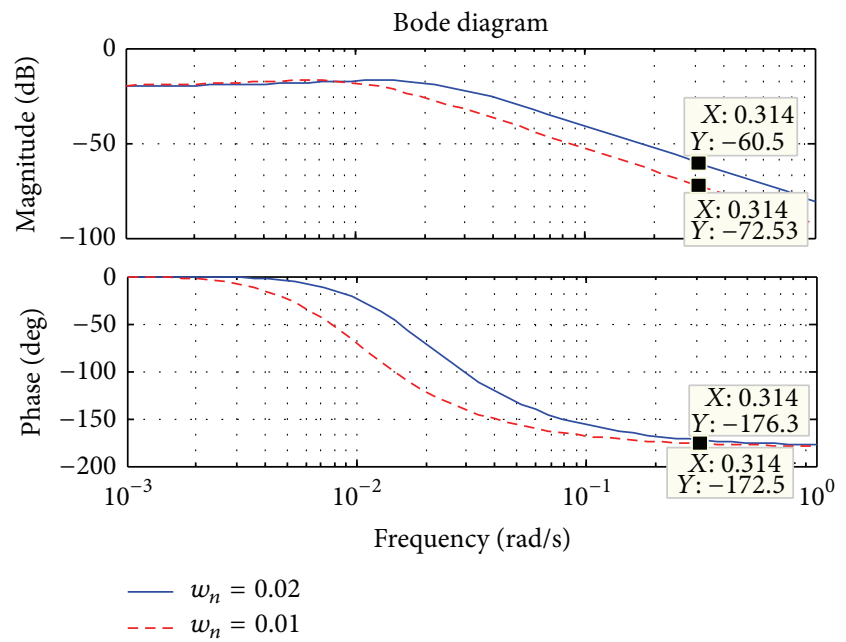

FIGURE 12: BODE plot of north acceleration to east misalignment.

$-4.2 \times 10^{-3}\left({ }^{\circ}\right),-4.5 \times 10^{-3}\left(^{\circ}\right)$, and $1.248\left(^{\circ}\right)$, that is nearly the same with the simulation results as well.

On one hand, the simulation result proves the performance of gyrocompass alignment on static base; on the other hand, it also proves the validity of error analysis for gyrocompass alignment on moving base discussed in Section 2.4. Therefore the error caused by carrier's movement has to be amended on moving base.

\subsection{Simulation Experiment of DVL Aided Gyrocompass Alignment on Moving Base}

5.2.1. The Simulation Conditions. Simulation experiments are proceeded in Harbin area, where the latitude $\varphi=45.7796^{\circ}$ 


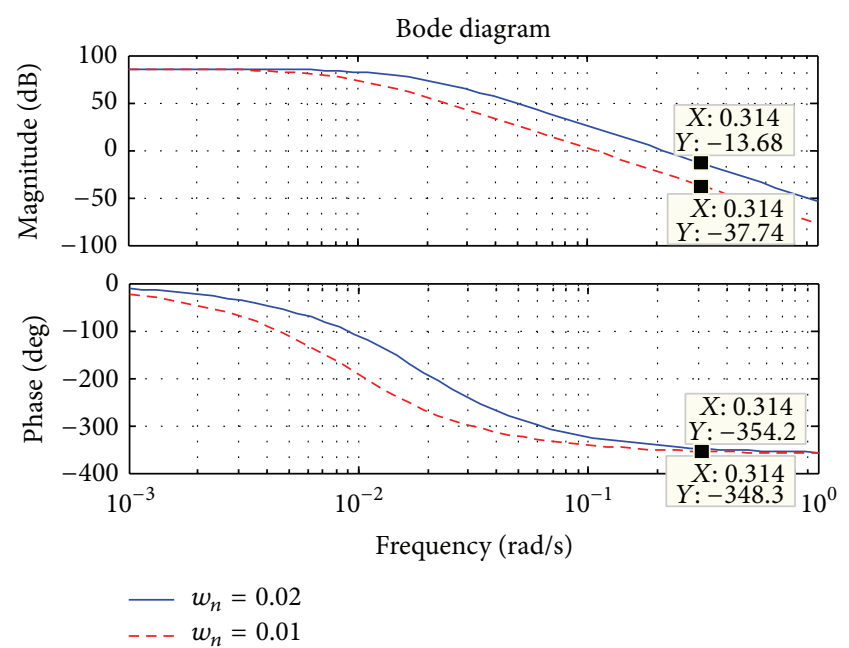

FIGURE 13: BODE plot of north acceleration to heading misalignment.

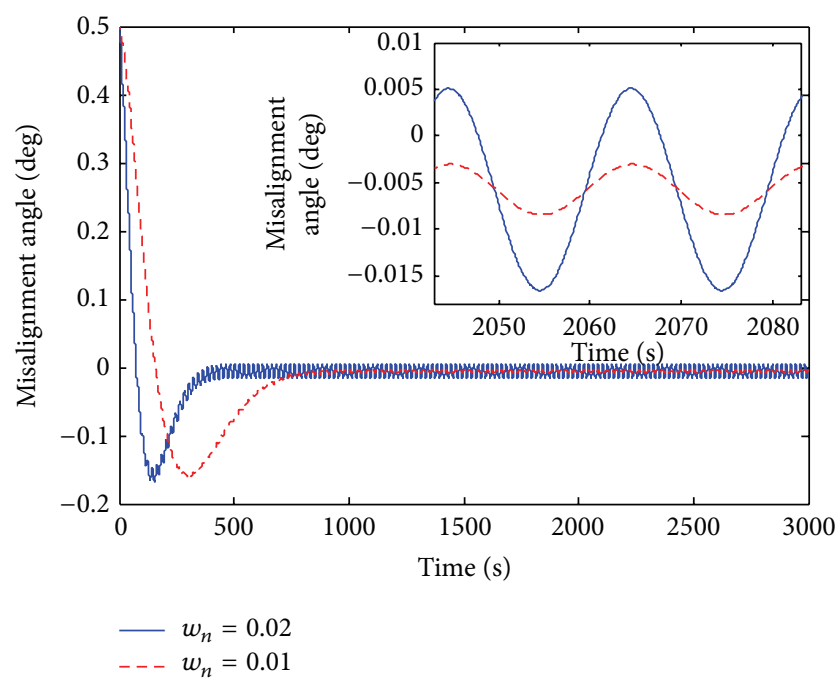

FIGURE 14: Curve of east misalignment caused by north acceleration.

and the longitude $\lambda=126.6705^{\circ}$. Ignoring all the other factors, the ship is assumed to sail along northeast direction. Set the initial velocity and gyro drift as zero and there exists acceleration on the gyrocompass alignment when the ship is moving. The period of acceleration oscillation is $20 \mathrm{~s}$ and its value is $A_{N}=A_{E}=0.2 \sin (2 \pi \cdot t / 20)$. Set the axis misalignment angles of coarse alignment as $0.5^{\circ}, 0.5^{\circ}$, and $0.5^{\circ}$, respectively.

5.2.2. The Simulation Results. We change $K$ value of the horizontal loop and the azimuth loop in which the damping ratio is still $\xi=0.8$ while oscillation frequency is adjusted from $\omega_{n}=0.02$ to $\omega_{n}=0.01$. In horizontal loop, $K_{1}=$ $0.0240, K_{2}=147.69$, and $K_{3}=0.5217$. In azimuth loop, $K_{1}=K_{3}=0.016, K_{2}=105.9534$, and $K_{4}=0.0042$. The whole simulation time is $3000 \mathrm{~s}$ and the simulation diagrams are drawn in Figures 12, 13, 14, and 15.

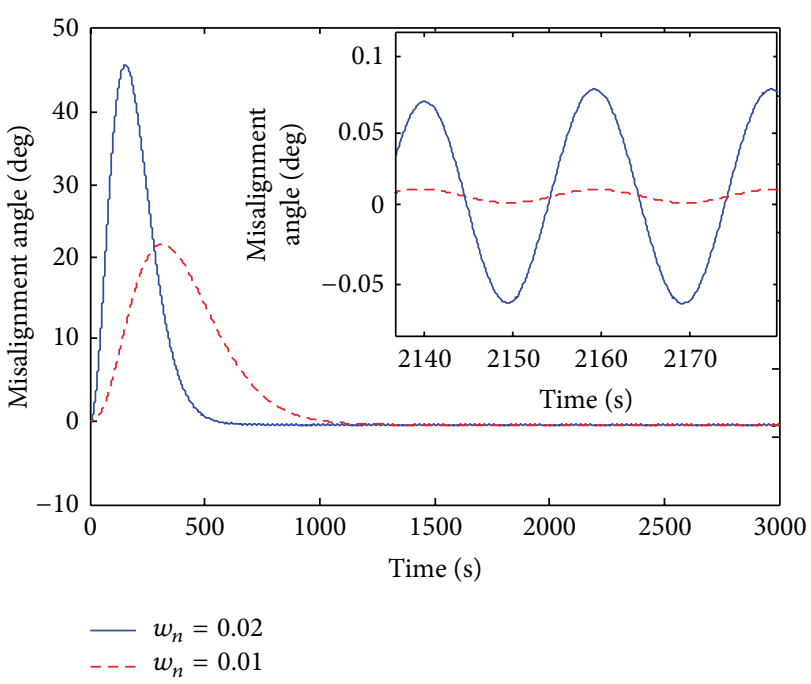

FIGURE 15: Curve of heading misalignment caused by north acceleration.

Figures $12-15$ show the BODE figure and corresponding misalignment curve before and after adjusting system parameters. At frequency of $0.314 \mathrm{rad} / \mathrm{sec}$ (the corresponding period is $20 \mathrm{~s}$ ), the magnitude is reduced from $-60.5 \mathrm{~dB}$ to $-72.5 \mathrm{~dB}$ in horizontal loop (as shown in Figure 12). Correspondingly the steady-state oscillation of misalignment is reduced from $0.6^{\prime}$ to around $0.18^{\prime}$ (as shown in Figure 14). In azimuth loop, the magnification is reduced from $-13.7 \mathrm{~dB}$ to $-37.7 \mathrm{~dB}$ (as shown in Figure 13), and the steady-state oscillation of misalignment is reduced from $4.5^{\prime}$ to around $0.5^{\prime}$ (as shown in Figure 15). It can be seen that the influence of acceleration can be effectively reduced by changing the parameters of system appropriately.

However, as shown in Figures 12-15, alignment time will also be prolonged accordingly. Therefore the data repeated calculation algorithm introduced in Section 4 is necessary, and it will efficiently shorten the alignment process.

\section{Test Verification}

6.1. Test Equipment Set-Up. To evaluate the performance of gyrocompass alignment deeply, a sailing test was conducted in testing field on Tai Lake (Wuxi, China). The test was conducted on a high-speed yacht platform equipped with several devices. It consists of an AHRS based on a fiber optical gyro (FOG) produced by our own research center (similar to [12], we conducted precalibration process of AHRS to minimize the error), a high-precise FOG-INS system called PHINS combined with GPS used as a reference system, and a DVL used to assist the AHRS system. Based on the heading and attitude information supplied by PHINS, the accuracy of gyrocompass initial alignment on moving base was evaluated. The characteristics of AHRS are shown in Table 2, and the performances of PHINS are shown in Table 3.

The high-speed yacht platform used in the test and the set-up of the equipment it carried are shown in Figure 16. 


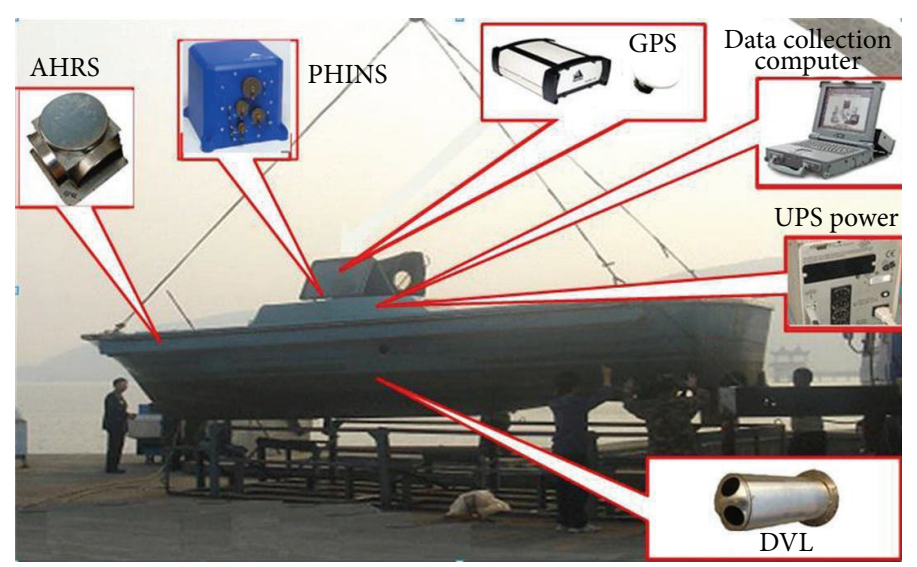

FIGURE 16: Yacht platform and the test equipment.

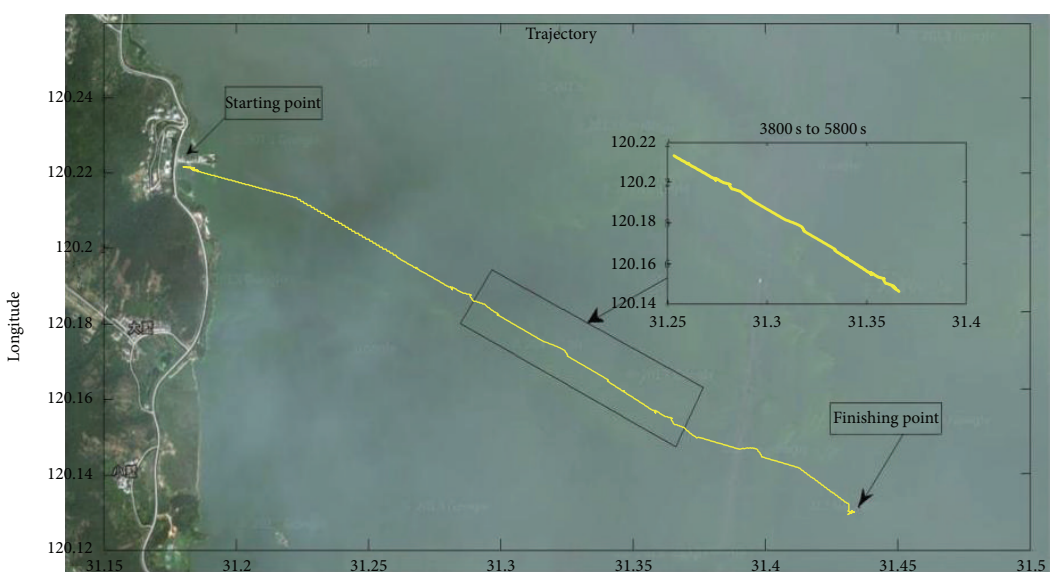

FIGURE 17: Test trajectory.

TABLE 2: The characteristics of AHRS.

\begin{tabular}{lclc}
\hline \multicolumn{2}{c}{ Gyroscope } & \multicolumn{2}{c}{ Accelerometers } \\
\hline $\begin{array}{l}\text { Bias-error } \\
\begin{array}{l}\text { Random walk } \\
\text { coefficient }\end{array}\end{array}$ & $0.01^{\circ} / \mathrm{h}$ & Threshold & $\pm 5 \times 10^{-5} \mathrm{~g}$ \\
$\begin{array}{l}\text { Scale factor } \\
\text { error }\end{array}$ & $<20 \mathrm{ppm}$ & $\begin{array}{l}\text { Scale factor } \\
\text { stability }\end{array}$ & $<100 \mathrm{ppm}$ \\
$\begin{array}{l}\text { Measuring } \\
\text { range }\end{array}$ & $\pm 55^{\circ} / \mathrm{s}$ & $\begin{array}{l}\text { Measuring } \\
\text { range }\end{array}$ & $\pm 15 \mathrm{~g}$ \\
\hline
\end{tabular}

6.2. Test Results and Analysis. The trajectory is across the testing field on Tai Lake. The yacht sailed for about 10 kilometers, and, by recording longitude and latitude of PHINS in real time, we can get the reference path of the yacht. Combined with the map of testing field, trajectory chart of the test is shown in Figure 17.

During the test, all the original data collected by gyros and accelerometers and other devices can be saved through data collecting system. The total time to complete the test is $8152 \mathrm{~s}$, and $2000 \mathrm{~s}$ (from $3800 \mathrm{~s}$ to $5800 \mathrm{~s}$ ) in the test is chosen

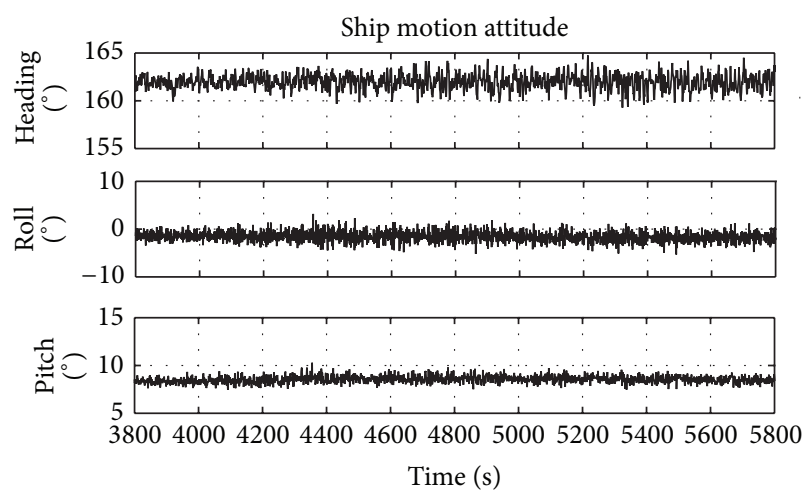

FIGURE 18: The ship's heading, roll, and pitch curves.

to conduct an off-line alignment simulation. Heading, roll, and pitch curves of the yacht are shown in Figure 18.

Through comparing the initial alignment results with PHINS synchronously, we can get the curves of heading and attitude error, as shown in Figure 19 (Method 1 corresponds 

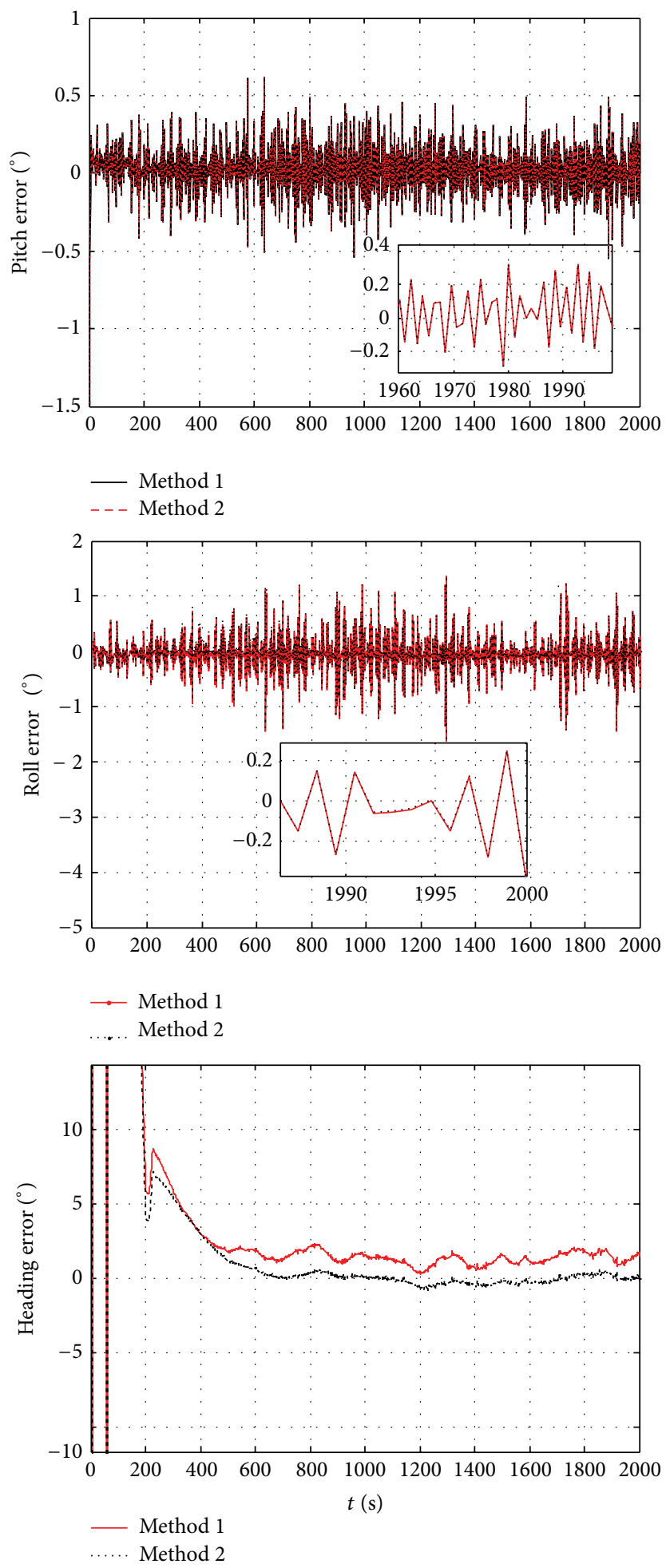

FIGURE 19: The restrain curves of heading and attitude error.

to the gyrocompass alignment algorithm on static base, while Method 2 corresponds to the gyrocompass alignment algorithm on moving base).

As shown in Figure 19, the pitch and roll error of gyrocompass alignment algorithm on moving base are of slight difference compared with algorithm on static base. But there is a significant difference in performance of heading angle on moving base. The error of algorithm on moving base is reduced to $1.5^{\circ}$ compared with that on static base. However, it still can be improved. As introduced in Section 3.2.3, while 
TABLE 3: The performance of PHINS.

\begin{tabular}{|c|c|c|c|c|c|}
\hline \multirow{2}{*}{$\begin{array}{l}\text { Position accuracy }(\text { CEP50\% } \\
\end{array}$} & \multicolumn{3}{|c|}{ Heading accuracy ( $1 \sigma$ value) } & \multicolumn{2}{|c|}{ Attitude accuracy ( $1 \sigma$ value) } \\
\hline & $5-15 \mathrm{~m}$ & With GPS aiding & $0.01^{\circ}$ secant latitude & Roll and pitch error & Less than $0.01^{\circ}$ \\
\hline With differential GPS aiding & $0.5-3 \mathrm{~m}$ & & & & \\
\hline With RTK differential GPS aiding & $2-5 \mathrm{~m}$ & No aiding & $0.05^{\circ}$ secant latitude & & \\
\hline No aiding for $5 \mathrm{~min}$ & $20 \mathrm{~m}$ & & & & \\
\hline Pure internal mode & $0.6 \mathrm{NM} / \mathrm{h}$ & & & & \\
\hline
\end{tabular}
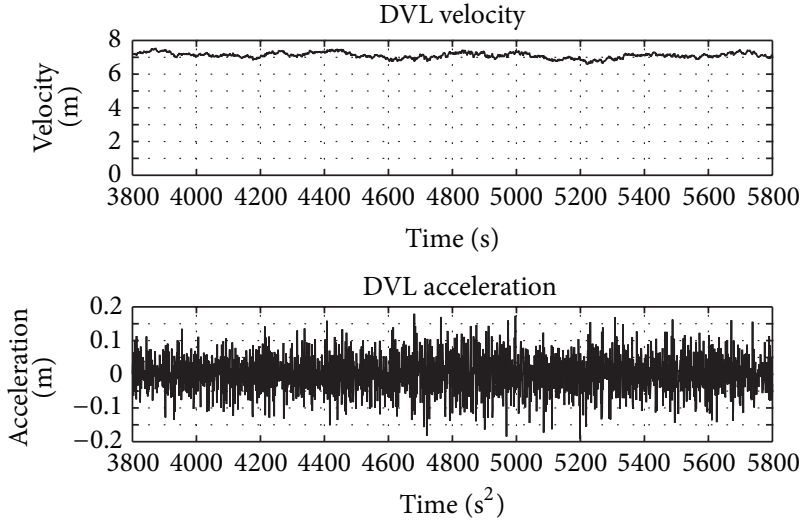

FIGURE 20: The velocity and acceleration curves under sailing condition.

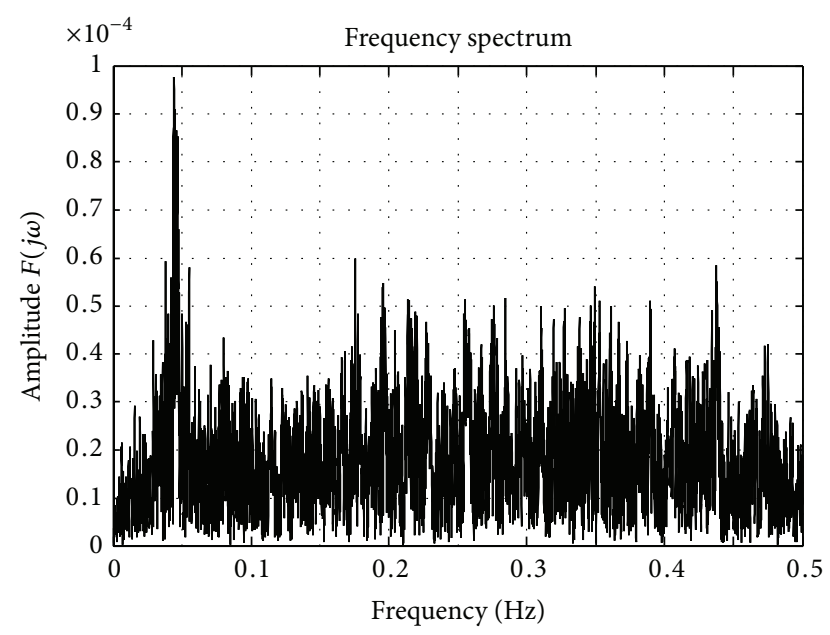

FIGURE 21: Frequency spectrogram of acceleration.

there exists a periodic interference acceleration in sailing condition, it will produce a periodic oscillation to heading. Inhibition of acceleration with this periodic oscillation can enhance the alignment performance further.

The velocity and acceleration curves of the yacht (from 3800 s to 5800 s) are shown in Figure 20.

In order to give a clear expression about how the acceleration affects the alignment system, a fast Fourier transform (FFT) is presented to the acceleration, and its frequency spectrogram is given in Figure 21.

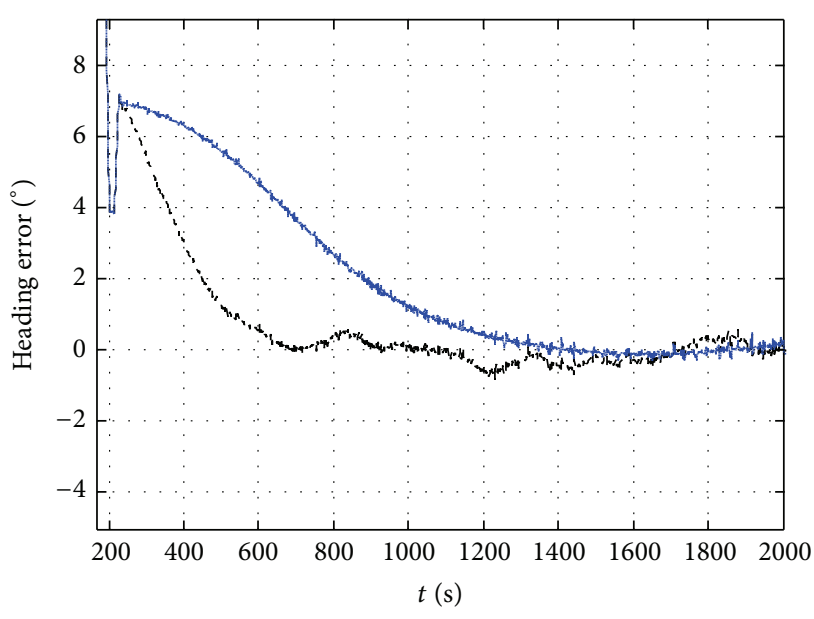

..... Method 2

-.. Method 3

FIGURE 22: Heading error restrain curves before and after reset.

Figure 21 provides a factor that there exists an oscillation period of $0.05 \mathrm{~Hz}$ in acceleration, and it will be equivalent to instrument error, which will seriously affect the result of heading alignment, so it is necessary to reset the alignment parameter of the gyrocompass loop.

The convergence curves are compared in Figure 22 (Method 2 corresponds to $\omega_{n}=0.05$, while Method 3 corresponds to $\omega_{n}=0.007$ ).

Curves in Figure 22 prove that, after reset of parameters, initial alignment results are much better than the former, but the alignment time is significantly increased from about $700 \mathrm{~s}$ to $1400 \mathrm{~s}$. To shorten the prolonged time, data repeated calculation algorithm is used, and the result is shown in Figure 23. In the first $220 \mathrm{~s}$, the coarse alignment process is carried out which can decrease the error angle to certain range quickly, but the precision cannot be guaranteed and the error vibration that causes a valley at nearly $200 \mathrm{~s}$ in Figure 23 is obvious. After $220 \mathrm{~s}$, the proposed alignment method is implemented after coarse alignment.

The chart in the middle represents the data repeated calculating process, which avoids the sampling step and almost takes less than $1 \mathrm{~s}$, so the alignment time is shortened to about $650 \mathrm{~s}$. After comparing the alignment curve in Figure 23 with that in Figure 22, it can be known that the alignment time is much shorter than before when the accuracy remains unchanged. 

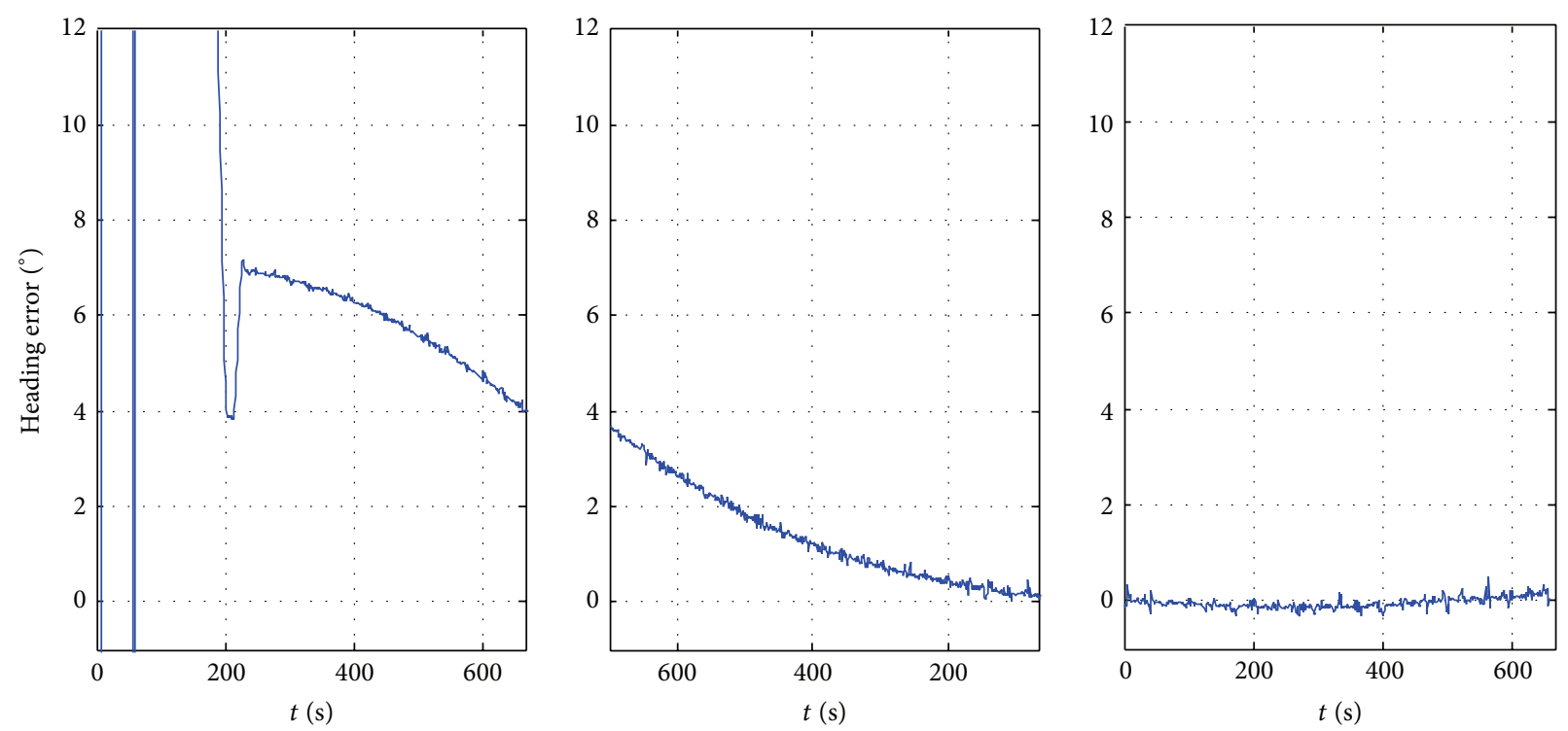

FIgURE 23: Time of heading alignment after using the data repeated calculation method.

TABLE 4: Statistics of 4 methods.

\begin{tabular}{lccccc}
\hline & & Method 1 & Method 2 & Method 3 & Method 4 \\
\hline Roll error & Mean & 0.0316 & 0.0314 & 0.0313 & 0.0313 \\
$\left(^{\prime}\right)$ & Variance & -0.1623 & -0.1618 & -0.1636 & -0.1636 \\
\hline Pitch error & Mean & 0.0646 & 0.0646 & 0.0645 & 0.0645 \\
$\left(^{\prime}\right)$ & Variance & 0.1107 & 0.1109 & 0.1105 & 0.1105 \\
\hline Yaw error & Mean & -1.4517 & -0.3507 & -0.1327 & -0.1327 \\
$\left(^{\circ}\right)$ & Variance & 0.3149 & 0.1791 & 0.1079 & 0.1079 \\
\hline \multicolumn{2}{l}{ Alignment time $(\mathrm{s})$} & 700 & 700 & 1400 & 650 \\
\hline \multicolumn{7}{l}{} \\
\end{tabular}

The experimental results of all four methods are put together in Table 4 in order to make a comparison. Method 1 is the gyrocompass alignment method using the algorithm on static base. Method 2 is the gyrocompass alignment method using the algorithm on moving base. Method 3 is the gyrocompass alignment method on moving base in which the control parameters are reset and Method 4 is the improvement of Method 3 after using the data repeated calculation algorithm. The mean and variance values in Table 4 are the statistics of error angle in the last $20 \mathrm{~s}$ of alignment process (from $1980 \mathrm{~s}$ to $2000 \mathrm{~s}$ ) comparing the alignment results with the standard value collected from PHINS.

From data in Table 4, it can be drawn that Method 4 has much better alignment results compared with the other three methods. That is to say, after using the gyrocompass alignment algorithm on moving base with resetting the system control parameters, the accuracy of initial alignment is guaranteed and the alignment time is also in acceptable range with the use of data repeated calculation algorithm.

\section{Conclusion}

Based on the principle analysis of classic platform initial gyrocompass alignment, a DVL aided gyrocompass alignment method for SINS on moving base is proposed in this paper. The implementation of algorithm is given and the influence of external velocity error is also analyzed. More specifically, two methods are adopted to cope with the gyrocompass alignment on moving base: first, an improved algorithm of gyrocompass alignment for SINS on moving base aided with DVL is introduced; then, after the error analysis, the system parameters are reset to decrease the acceleration interference. However, from results it turns out that alignment time is too long to be accepted. Aiming at this problem, a data repeated calculation algorithm is put forward to shorten the prolonged time. The simulation and experimental results verify the performance of the proposed alignment method both in accuracy and convergence time.

\section{Abbreviations}

$R: \quad$ Earth radius

$g$ : $\quad$ Gravitational acceleration

$\omega_{i e}$ : Rotational angular velocity of the earth

$\phi_{x}, \phi_{y}, \phi_{z}$ : The east, north, and azimuth misalignment angle of platform

$\varphi: \quad$ Latitude

$\lambda$ : $\quad$ Longitude

$t: \quad$ The geographical coordinates

$n: \quad$ The navigation coordinates

p: $\quad$ The platform coordinates

$b$ : The body coordinates

$i$ : The geocentric inertial coordinates

$e$ : The earth coordinates 


\begin{tabular}{|c|c|}
\hline$C_{b}^{p}:$ & $\begin{array}{l}\text { The transform matrix from the platform } \\
\text { frame } p \text { to the SINS's body frame } b\end{array}$ \\
\hline$\omega_{p b}^{b}:$ & $\begin{array}{l}\text { Angular rate of the body frame with } \\
\text { respect to the platform frame }\end{array}$ \\
\hline & The control angular rate \\
\hline & $\begin{array}{l}\text { The angular rate of the body frame with } \\
\text { respect to the inertial frame }\end{array}$ \\
\hline & \\
\hline & bias \\
\hline & ng coefficient of the system \\
\hline & \\
\hline & ncy \\
\hline$\phi_{s x}, \phi_{s y}, \phi_{s z}$ & caused by device error in \\
\hline & Heading \\
\hline & $\begin{array}{l}\text { e directly measured by the } \\
\text { ody frame }\end{array}$ \\
\hline & $\begin{array}{l}\text { Specific force directly measured by the } \\
\text { IMU in the navigation frame }\end{array}$ \\
\hline$B^{p}:$ & Bad acceleration \\
\hline$\varepsilon_{d e}, \varepsilon_{d n}, \varepsilon_{d u}$ & $\begin{array}{l}\text { drift on uniform } \\
\text { ons }\end{array}$ \\
\hline$\nabla_{d e}, \nabla_{d n}, \nabla_{d u}$ & $\begin{array}{l}\text { The equivalent accelerometer bias on } \\
\text { uniform motion in three directions }\end{array}$ \\
\hline & The speed of body measured by DVL \\
\hline & $\begin{array}{l}\text { The error angle caused in three direc- } \\
\text { tions by uniform motion }\end{array}$ \\
\hline$\varepsilon_{\text {Ade }}, \varepsilon_{A d n}, \varepsilon_{A d u}:$ & $\begin{array}{l}\text { The equivalent gyro drift in three direc- } \\
\text { tions on accelerated movement }\end{array}$ \\
\hline$\nabla_{\text {Adn }}, \nabla_{\text {Ade }}, \nabla_{\text {Adu }}:$ & $\begin{array}{l}\text { The equivalent accelerometer bias } \\
\text { on accelerated movement in three } \\
\text { directions }\end{array}$ \\
\hline$A, A, A$ & $\begin{array}{l}\text { The error angle caused by accelerated } \\
\text { movement in three directions. }\end{array}$ \\
\hline
\end{tabular}

\section{Conflict of Interests}

The authors declare that there is no conflict of interests regarding the publication of this paper.

\section{Acknowledgments}

This study is supported in part by the National Natural Science Foundation of China (Grant no. 61203225), the State Postdoctoral Science Foundation (2012M510083), and the Central college Fundamental Research Special Fund (no. HEUCF110427). The authors would like to thank the anonymous reviewers for their constructive suggestions and insightful comments.

\section{References}

[1] E. Levinson and C. S. Giovanni, "Laser gyro potential for long endurance marine navigation," in Proceedings of the IEEE Position Location and Navigation Symposium, pp. 115-129, Piscataway, NJ, USA, December 1980.

[2] P. M. G. Silson, "Coarse alignment of a ship's strapdown inertial attitude reference system using velocity loci," IEEE Transactions on Instrumentation and Measurement, vol. 60, no. 6, pp. 19301941, 2011.

[3] J. C. Yu, J. B. Chen, and J. H. Han, "Multiposition observability analysis of strapdown inertial navigation system," Transaction of Beijing Institute of Technology, vol. 24, no. 2, pp. 150-153, 2004 (Chinese).

[4] X. L. Wang and G. X. Sheng, "Fast and precision multiposition initial alignment method of inertial navigation system," Journal of Astronautics, vol. 23, no. 4, pp. 81-84, 2002 (Chinese).

[5] P. M. Lee, B. H. Jun, K. Kim, J. Lee, T. Aoki, and T. Hyakudome, "Simulation of an inertial acoustic navigation system with range aiding for an autonomous underwater vehicle," IEEE Journal of Oceanic Engineering, vol. 32, no. 2, pp. 312-345, 2007.

[6] S. S. Gao, W. H. Wei, Y. Zhong, and Z. Feng, "Rapid alignment method based on local observability analysis for strapdown inertial navigation system," Acta Astronautica, vol. 94, no. 2, pp. 790-793, 2014.

[7] T. Zhang and X. S. Xu, "A new method of seamless land navigation for GPS/INS integrated system," Measurement, vol. 45, no. 4, pp. 691-701, 2012.

[8] X. X. Liu, X. S. Xu, Y. T. Liu, and L. H. Wang, "A fast and high-accuracy compass alignment method to sins with azimuth axis rotation," Mathematical Problems in Engineering, vol. 2013, Article ID 524284, 12 pages, 2013.

[9] X. H. Cheng and M. Zheng, "Optimization on Kalman filter parameters of SINS during initial alignment," Journal of Chinese Inertial Technology, vol. 14, no. 4, pp. 15-16, 2006 (Chinese).

[10] G. M. Yan, W. S. Yan, and D. M. Xu, "On reverse navigation algorithm and its application to SINS gyro-compass in-movement alignment," in Proceedings of the 27th Chinese Control Conference (CCC '08), pp. 724-729, Kunming, China, July 2008.

[11] X. X. Liu, X. S. Xu, Y. T. Liu, and L. H. Wang, "A rapid transfer alignment method for SINS based on the added backwardforward SINS resolution and data fusion," Mathematical Problems in Engineering, vol. 2013, Article ID 401794, 10 pages, 2013.

[12] J. M. Hwang, S. S. Han, and J. M. Lee, "Applications of dualelectric compasses to spreader pose control," International Journal of Control, Automation and Systems, vol. 8, no. 2, pp. 433-438, 2010.

[13] D. H. Titterton and J. L. Weston, Strapdown Inertial Navigation Technology, Institute of Electrical Engineers, Hampshire, UK, 2nd edition, 2004.

[14] Y. Yang and L. J. Miao, "Fiber-optic strapdown inertial system with sensing cluster continuous rotation," IEEE Transactions on Aerospace and Electronic Systems, vol. 40, no. 4, pp. 1173-1178, 2004.

[15] Y. N. Gao, J. B. Chen, and T. P. Yang, "Error analysis of strapdown optic fiber gyro compass," Transaction of Beijing Institute of Technology, vol. 25, no. 5, pp. 423-426, 2005.

[16] J. C. Fang and D. J. Wan, "A fast initial alignment method for strapdown inertial navigation system on stationary base," IEEE Transactions on Aerospace and Electronic Systems, vol. 32, no. 4, pp. 1501-1505, 1996.

[17] X. Liu and X. Xu, "System calibration techniques for inertial measurement units," Journal of Chinese Inertial Technology, vol. 17, no. 5, pp. 568-571, 2009 (Chinese).

[18] A. Chatfield, Fundamentals of High Accuracy Inertial Navigation, Institute of Astronautics and Aeronautics, Reston, Va, USA, 1997. 
[19] Y. Li, X. X. Xu, and B. X. Wu, "Gyrocompass self-alignment of SINS," Journal of Chinese Inertial Technology, vol. 16, no. 4, pp. 386-389, 2008 (Chinese).

[20] R. McEwen, H. Thomas, D. Weber, and F. Psota, "Performance of an AUV navigation system at arctic latitudes," IEEE Journal of Oceanic Engineering, vol. 30, no. 2, pp. 443-454, 2005. 


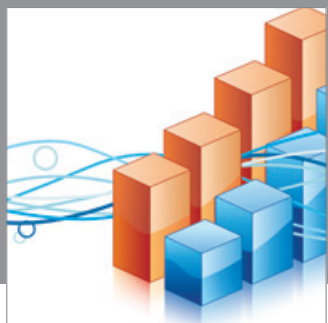

Advances in

Operations Research

mansans

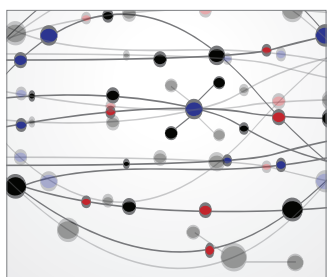

The Scientific World Journal
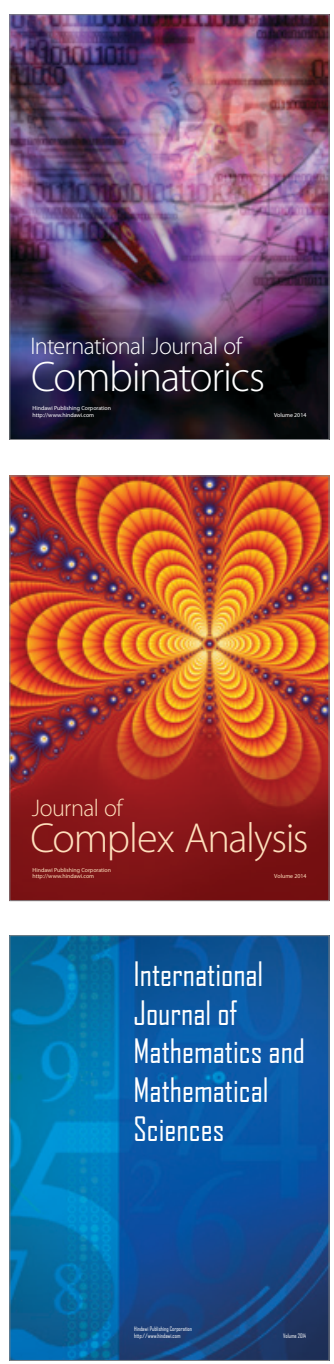
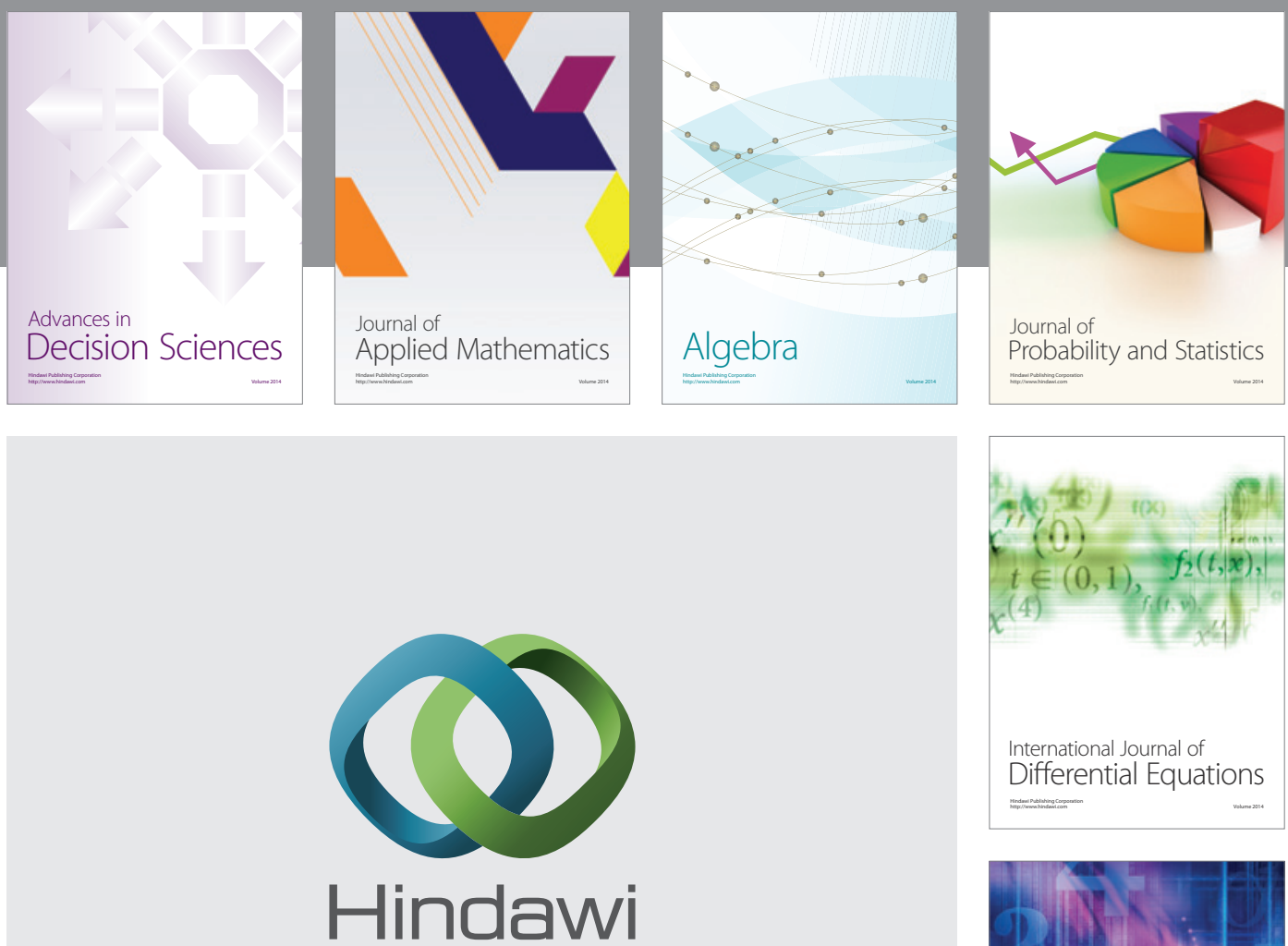

Submit your manuscripts at http://www.hindawi.com
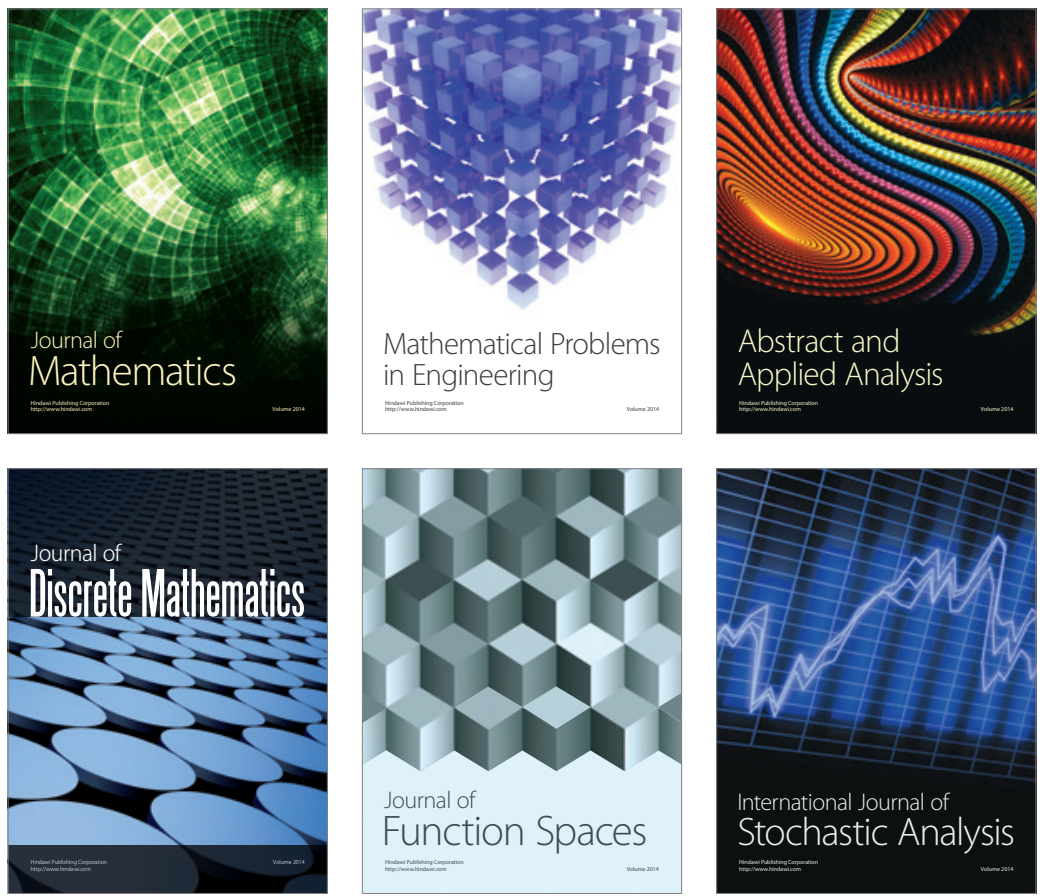

Journal of

Function Spaces

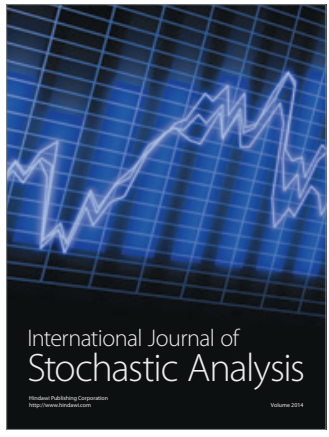

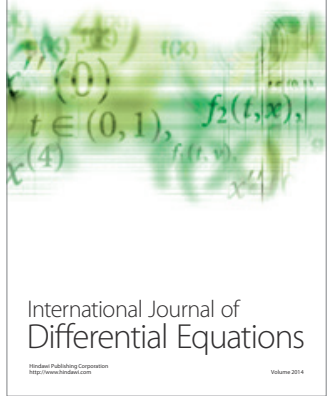
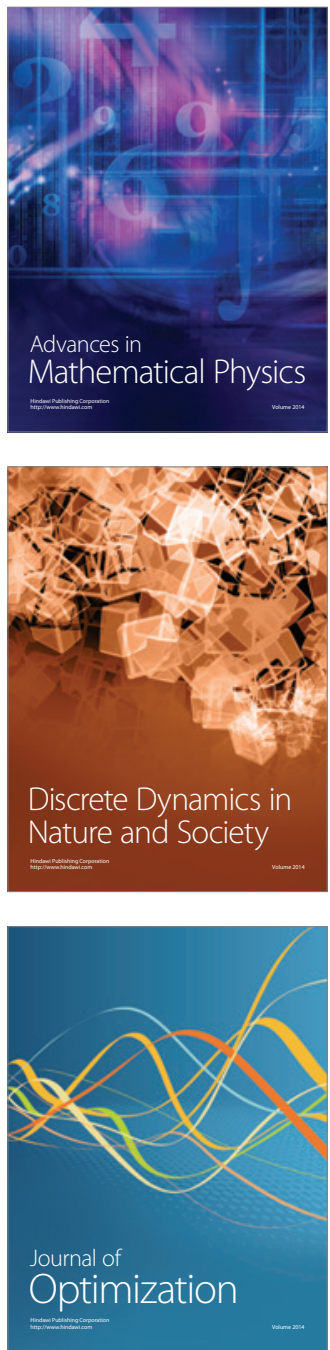